\title{
Growth and yield performance of mutant ginger (Zingiber officinale Rosc.) lines in South-Eastern Nigeria
}

\author{
Mary N. Abua ${ }^{1}$, Godfrey A. Iwo ${ }^{1}$, Macauley A. Ittah ${ }^{1}$, Ekemini E. Obok ${ }^{1 *}$ (D), Richmond E. Edugbo ${ }^{2}$ \\ ${ }^{1}$ Department of Crop Science, Faculty of Agriculture, Forestry and Wildlife Resources Management, University of Calabar, Calabar, Nigeria. \\ ${ }^{2}$ Department of Agronomy, Michael Okpara University of Agriculture, Umuahia, Nigeria.
}

\begin{tabular}{l}
\hline ARTICLE INFO \\
\hline Article history: \\
Received on: January 12, 2021 \\
Accepted on: May 27, 2021 \\
Available online: September 01, 2021 \\
\hline
\end{tabular}

Key words:

Gamma-ray, ginger, irradiation, landraces, rhizome, mutation, spices

\begin{abstract}
A field evaluation on growth and yield performances of 15 mutant lines and two landraces of Zingiber officinale (Rosc.) was conducted in Cross River State, Nigeria, in 2016 and 2017. The experiment was laid out in a Randomized Complete Block Design (RCBD) with three replications in each of the three locations, Calabar, Ikom, and Ogoja. Combined analysis of variance showed significant $(p<0.05)$ growth and yield differences among the 17 ginger genotypes. Nine mutant lines, UG1-5-04, UG1-5-35, UG2-9-01, UG1-13-02, UG1-7-24, UG1-5-38, UG1-5-31, UG2-11-03, and UG1-5-18, had superior rhizome yield ranging from 18.44 to $22.06 \mathrm{t} /$ ha and were significantly different $(p>0.05)$ from the two landraces, UG1 $(14.39 \mathrm{t} / \mathrm{ha})$ and UG2 $(14.72 \mathrm{t} / \mathrm{ha})$. Mutant UG2-9-01 had the highest average number of rhizomes per plant (21.44) and the longest rhizomes (20.46 $\mathrm{cm})$. Mutant UG1-5-04 had the highest total rhizome yield per hectare (22.06 t/ha). The overall performance of the nine mutant ginger lines across the 2 years was superior and similar $(p<0.05)$ in Ogoja and Ikom locations in comparison with Calabar location. The two locations, Ikom and Ogoja, were recommended as the most suitable environments for the cultivation of the nine promising mutant lines of ginger in Cross River State.
\end{abstract}

\section{INTRODUCTION}

Ginger (Zingiber officinale Rosc.) is an important spice in the family Zingiberaceae with 90 species [1]. Ginger plant is refreshingly aromatic but it is the rhizome (raw or processed) that is valued as a spice. Ginger is produced in more than 25 countries in the world. Nigeria, Nepal, India, China, Indonesia, Thailand, Korea, Philippines, Australia, and Malaysia are the major growing countries. Ginger as a spice is produced on a large scale in Nigeria for export compared to other spices like garlic, onion, and pepper and highly valued in the international market because of its aroma, pungency, and oleoresin content [2]. Ginger is extremely important in the production of curry powder and gingerbread and in some beers and other beverages. In addition to their medicinal qualities, ginger extracts may also serve as a natural larvicidal agent [3]. The refreshing aroma with strong taste makes ginger an important ingredient of most world food processing industries [4].

\footnotetext{
*Corresponding Author

Ekemini Edet Obok, Department of Crop Science, Faculty of Agriculture,

Forestry and Wildlife Resources Management, University of Calabar,

Calabar, Nigeria.E-mail:e.e.obok@unical.edu.ng
}

The production trend of ginger in Nigeria is low when compared to other export crops due to its poor yields which can be attributed to the lack of improved varieties [5]. In spite of these poor yields, Nigeria is the main producer and exporter of ginger in Africa and ranks 4th in the world after India, China, and Nepal [6]. The average production of ginger in Nigeria annually is 50,000 metric tonnes [7], out of which about $10 \%$ is used locally as fresh ginger while $90 \%$ is processed for both local usage and export. Owing to its status as a minor crop, ginger attracts little research effort; as a result, the yields have remained generally low due to lack of improved varieties [8].

The major constraint to ginger production in Nigeria is narrow gene pool, poor flowering, and lack of seed set [1]. Unsuitable soils and unfavorable ecological factors as well as lack of improved varieties are reasons for low yield and productivity [9]. Over 60 years of ginger cultivation in Nigeria, farmers have relied entirely on two local landraces, namely, UG1 and UG2 [10]. This lack of improved varieties has resulted in the low yields obtained by ginger farmers in Nigeria. However, new ginger lines have been developed through mutation breeding; these mutant lines must be evaluated in different agroecological zones to determine 
their growth and yield performance, adaptation, and superiority before they are released for wide cultivation. This has prompted this research across three locations in Cross River State, Nigeria. Thus, the objective of the study was a 2-year (2016 and 2017) field evaluation of growth and yield performances of 15 mutant lines and two landraces of ginger at three geographically distinct locations (Calabar, Ikom, and Ogoja) in Cross River State, Nigeria.

\section{MATERIALS AND METHODS}

\subsection{Experimental Site}

This experiment was carried out across three locations in Cross River State, Nigeria, during the 2016 and 2017 cropping seasons (March to December). The locations were Calabar $\left(4.9757^{\circ}\right.$ $\mathrm{N}, 8.3417^{\circ} \mathrm{E}$ ) (soil $\mathrm{pH}: 5.7$, effective cation exchange capacity (ECEC): 7.94, and soil texture: loamy sand), usually with an annual rainfall of 2,915-3,500 $\mathrm{mm}$ and optimum temperature of $26^{\circ} \mathrm{C}$, Ikom $\left(5.9617^{\circ} \mathrm{N}, 8.7206^{\circ} \mathrm{E}\right.$ ) (soil pH: 5.8, ECEC: 11.74, and soil texture: sandy clay loam), with an annual rainfall of 2,250-2,332 $\mathrm{mm}$ and optimum temperature of $27^{\circ} \mathrm{C}$, and Ogoja $\left(6.6548^{\circ} \mathrm{N}, 8.7977^{\circ} \mathrm{E}\right)$ (soil $\mathrm{pH}: 5.2, \mathrm{ECEC}: 5.57$, and soil texture: sandy loam), with an annual rainfall of 1,848-2,200 $\mathrm{mm}$ and optimum temperature of $28.7^{\circ} \mathrm{C}$ [11]. The three locations were previously cropped to leafy vegetables under conventional organic farming management. Tables $1-3$ give an overview of the actual rainfall, sunshine, temperature, and relative humidity recordings at the three experimental sites during the study period.

\subsection{Planting Materials and Source}

Seventeen ginger genotypes consisting of fifteen (15) mutant lines (UG1-11-07, UG1-13-02, UG1-2-35, UG1-5-04, UG1-5-18, UG1-5-22, UG1-5-31, UG1-5-35, UG1-5-38, UG1-5-48, UG1-5-
49, UG1-5-52, UG1-7-24, UG2-11-03, and UG2-9-01) and two local check landraces (UG1 and UG2) were sourced from National Root Crop Research Institute (NRCRI), Umudike, Nigeria. The 15 mutant lines were derived from the existing landraces UG1 and UG2 by exposing them to different doses of gamma-ray irradiation. The mutant lines derived from UG1 were exposed to $2 \mathrm{GY}, 5 \mathrm{GY}, 7 \mathrm{GY}, 11 \mathrm{GY}$, and $13 \mathrm{GY}$ doses of gamma-ray to give the following mutant lines: UG1-2-35, UG1-5-04, UG1-5-18, UG15-22, UG1-5-31, UG1-5-35, UG1-5-38, UG1-5-48, UG1-5-49, UG1-5-52, UG1-7-24, UG1-11-07, and UG1-13-02. The mutant lines derived from UG2 were exposed to $9 \mathrm{GY}$ and $11 \mathrm{GY}$ doses of gamma-ray to give the following mutant lines: UG1-9-01 and UG2-11-03.

\subsection{Field Layout, Experimental Design, and Data Collection}

This experiment was a split plot laid out in a Randomized Complete Block Design with three replications. An experimental plot measuring $26 \times 8 \mathrm{~m}\left(208 \mathrm{~m}^{2}\right)$ was used for this research in each of the locations. Each experimental unit measured $1 \times 2 \mathrm{~m}$ $\left(2 \mathrm{~m}^{2}\right)$ with $0.5 \mathrm{~m}$ alley. The ginger setts or rhizomes weighing 10 $\mathrm{g}$ were planted in rows with inter- and intrarow spacing of $50 \mathrm{~cm}$ giving a plant population density of $40,000 \mathrm{~kg} / \mathrm{ha}$. Each rhizome was planted $4-5 \mathrm{~cm}$ beneath the soil with the growth buds facing up so that the shoots can grow towards the surface. Mulching was carried out immediately after planting using Chromolaena odorata (L.) R. M. King \& H. Rob (commonly called Siam weed). Suitable agronomic practices were carried out. Nitrogen-PhosphorusPotassium 15-15-15 fertilizer was applied at $120 \mathrm{kgN} / \mathrm{ha}$ in a split dose of 80 and $40 \mathrm{~kg}$ at 2 and 6 weeks after planting. Weeds were manually controlled at 2 and 6 weeks after planting (before fertilizer application). The fields were under rainfed irrigation. Data were collected on the following traits during growth and development: sprouting percentage $(\%)$, establishment count $(\%)$,

Table 1. Monthly weather conditions at Ikom in 2016 and 2017 (January-December).

\begin{tabular}{|c|c|c|c|c|c|c|c|c|c|c|}
\hline \multirow[t]{2}{*}{ Month } & \multicolumn{2}{|c|}{ Rainfall (mm) } & \multicolumn{2}{|c|}{ Sunshine (hours) } & \multicolumn{2}{|c|}{$\operatorname{Tmax}\left({ }^{\circ} \mathrm{C}\right)$} & \multicolumn{2}{|c|}{$\operatorname{Tmin}\left({ }^{\circ} \mathrm{C}\right)$} & \multicolumn{2}{|c|}{ RH (\%) } \\
\hline & 2016 & 2017 & 2016 & 2017 & 2016 & 2017 & 2016 & 2017 & 2016 & 2017 \\
\hline January & 0.0 & 49.9 & 7.43 & 7.32 & 35.1 & 34.1 & 20.0 & 22.0 & 68 & 75 \\
\hline February & 7.4 & 0.0 & 6.11 & 6.81 & 37.4 & 35.2 & 23.3 & 20.9 & 72 & 71 \\
\hline March & 131.5 & 64.3 & 5.08 & 6.80 & 33.8 & 36.7 & 24.7 & 25.3 & 83 & 76 \\
\hline April & 157.7 & 210.3 & 6.07 & 6.69 & 34.2 & 33.5 & 24.7 & 24.0 & 83 & 82 \\
\hline May & 383.7 & 247.6 & 7.04 & 6.47 & 32.9 & 32.3 & 23.6 & 23.6 & 83 & 86 \\
\hline June & 402.5 & 223.8 & 5.20 & 5.90 & 31.5 & 31.5 & 23.6 & 23.3 & 87 & 87 \\
\hline July & 538.7 & 329.0 & 4.22 & 3.63 & 30.2 & 29.8 & 23.0 & 23.4 & 92 & 91 \\
\hline August & 422.8 & 407.1 & 2.33 & 2.02 & 30.1 & 28.7 & 23.4 & 22.7 & 92 & 91 \\
\hline September & 234.3 & 343.3 & 3.28 & 3.05 & 31.0 & 30.3 & 20.5 & 23.2 & 91 & 87 \\
\hline October & 236.5 & 328.1 & 6.37 & 4.36 & 32.6 & 31.6 & 22.9 & 23.5 & 86 & 84 \\
\hline November & 9.4 & 66.3 & 7.56 & 4.54 & 34.0 & 32.5 & 23.4 & 23.3 & 83 & 83 \\
\hline December & 0.0 & 4.6 & 6.78 & 4.22 & 33.9 & 33.7 & 22.3 & 21.4 & 77 & 76 \\
\hline Total & $2,524.5$ & $2,274.3$ & 67.5 & 61.8 & - & - & - & - & - & - \\
\hline Mean & 210.38 & 189.53 & 5.62 & 5.15 & 33.06 & 32.49 & 22.95 & 23.05 & 83.08 & 82.42 \\
\hline$t_{\mathrm{df}=11 ; \alpha=0.05}$ & \multicolumn{2}{|c|}{ NS } & \multicolumn{2}{|c|}{ NS } & \multicolumn{2}{|c|}{ NS } & \multicolumn{2}{|c|}{ NS } & \multicolumn{2}{|c|}{ NS } \\
\hline
\end{tabular}

NS: paired student's $T$-test of the mean weather condition for the 2 years is not significant at $95 \%$ confidence level

${ }^{a}$ Calculation of cumulative value (i.e., total) not applicable. Thus, the use of dash. 
Table 2. Monthly weather conditions at calabar in 2016 and 2017 (January-December).

\begin{tabular}{|c|c|c|c|c|c|c|c|c|c|c|}
\hline \multirow{2}{*}{ Month } & \multicolumn{2}{|c|}{ Rainfall (mm) } & \multicolumn{2}{|c|}{ Sunshine (hours) } & \multicolumn{2}{|c|}{$\operatorname{Tmax}\left({ }^{\circ} \mathrm{C}\right)$} & \multicolumn{2}{|c|}{$\operatorname{Tmin}\left({ }^{\circ} \mathrm{C}\right)$} & \multicolumn{2}{|c|}{ RH (\%) } \\
\hline & 2016 & 2017 & 2016 & 2017 & 2016 & 2017 & 2016 & 2017 & 2016 & 2017 \\
\hline January & 0.0 & 25.4 & 5.10 & 4.30 & 33.7 & 33.4 & 22.9 & 23.4 & 69 & 82 \\
\hline February & 3.5 & 0.0 & 5.30 & 5.60 & 35.2 & 33.9 & 24.7 & 24.0 & 78 & 82 \\
\hline March & 194.9 & 133.7 & 4.50 & 4.00 & 32.6 & 33.2 & 24.0 & 24.7 & 87 & 83 \\
\hline April & 203.4 & 417.2 & 4.20 & 4.80 & 32.4 & 32.3 & 24.2 & 23.3 & 87 & 86 \\
\hline May & 421.1 & 284.7 & 4.60 & 4.70 & 32.1 & 31.3 & 23.8 & 23.0 & 86 & 88 \\
\hline June & 261.0 & 352.2 & 2.70 & 3.60 & 30.6 & 30.8 & 22.7 & 23.0 & 88 & 89 \\
\hline July & 445.6 & 437.2 & 1.70 & 1.60 & 29.3 & 29.0 & 22.6 & 22.8 & 91 & 91 \\
\hline August & 299.2 & 611.4 & 1.10 & 1.10 & 28.7 & 28.2 & 22.8 & 22.7 & 93 & 94 \\
\hline September & 363.3 & 356.0 & 2.10 & 1.80 & 29.3 & 29.0 & 23.1 & 23.4 & 91 & 90 \\
\hline October & 95.1 & 180.3 & 2.10 & 2.90 & 30.9 & 30.4 & 23.0 & 23.4 & 87 & 88 \\
\hline November & 1.2 & 289.2 & 5.20 & 4.60 & 31.9 & 31.4 & 23.6 & 23.4 & 87 & 88 \\
\hline December & 0.6 & 32.3 & 4.70 & 4.00 & 33.2 & 32.5 & 22.8 & 23.9 & 83 & 81 \\
\hline Total & $2,288.9$ & $3,119.6$ & 43.3 & 43.0 & $-{ }^{\mathrm{a}}$ & $-{ }^{\mathrm{a}}$ & $--^{\mathrm{a}}$ & $-{ }^{\mathrm{a}}$ & $--^{\mathrm{a}}$ & $-{ }^{\mathrm{a}}$ \\
\hline Mean & 190.74 & 259.97 & 3.61 & 3.58 & 31.66 & 31.28 & 23.35 & 23.42 & 85.58 & 86.83 \\
\hline$t_{\mathrm{df}=11 ; \alpha=0.05}$ & \multicolumn{2}{|c|}{ NS } & \multicolumn{2}{|c|}{ NS } & \multicolumn{2}{|c|}{ NS } & \multicolumn{2}{|c|}{ NS } & \multicolumn{2}{|c|}{ NS } \\
\hline
\end{tabular}

NS =: paired student's $t$-test of the mean weather condition for the 2 years is not significant at $95 \%$ confidence level.

${ }^{a}$ :cCalculation of cumulative value (i.e., total) not applicable.

Table 3. Monthly weather conditions at Ogoja in 2016 and 2017 (January-December).

\begin{tabular}{|c|c|c|c|c|c|c|c|c|c|c|}
\hline \multirow{2}{*}{ Month } & \multicolumn{2}{|c|}{ Rainfall (mm) } & \multicolumn{2}{|c|}{ Sunshine (hours) } & \multicolumn{2}{|c|}{$\operatorname{Tmax}\left({ }^{\circ} \mathrm{C}\right)$} & \multicolumn{2}{|c|}{$\operatorname{Tmin}\left({ }^{\circ} \mathrm{C}\right)$} & \multicolumn{2}{|c|}{ RH (\%) } \\
\hline & 2016 & 2017 & 2016 & 2017 & 2016 & 2017 & 2016 & 2017 & 2016 & 2017 \\
\hline January & 0.0 & 3.2 & 8.10 & 7.20 & 36.1 & 36.0 & 18.3 & 21.8 & 33 & 54 \\
\hline February & 0.0 & 0.0 & 9.00 & 7.80 & 35.8 & 37.5 & 22.6 & 20.2 & 48 & 35 \\
\hline March & 44.9 & 0.0 & 7.60 & 5.70 & 35.3 & 38.5 & 25.3 & 25.6 & 72 & 64 \\
\hline April & 126.4 & 154.7 & 5.80 & 5.60 & 33.4 & 35.5 & 25.0 & 24.6 & 74 & 71 \\
\hline May & 209.3 & 362.1 & 6.20 & 3.80 & 33.3 & 38.3 & 23.9 & 23.5 & 79 & 78 \\
\hline June & 167.1 & 152.5 & 3.40 & 3.00 & 32.3 & 32.4 & 23.4 & 23.5 & 80 & 80 \\
\hline July & 309.2 & 164.6 & 3.00 & 4.80 & 30.7 & 31.2 & 23.1 & 23.3 & 85 & 84 \\
\hline August & 333.1 & 367.6 & 3.20 & 4.00 & 30.8 & 30.1 & 23.4 & 23.0 & 86 & 85 \\
\hline September & 255.8 & 285.8 & 5.00 & 3.50 & 32.7 & 31.0 & 23.4 & 22.9 & 84 & 83 \\
\hline October & 288.0 & 222.5 & 4.80 & 4.20 & 32.6 & 32.6 & 23.9 & 23.5 & 80 & 80 \\
\hline November & 25.9 & 46.1 & 8.00 & 4.70 & 33.4 & 34.2 & 24.0 & 23.0 & 77 & 79 \\
\hline December & 0.0 & 0.0 & 6.80 & 5.00 & 35.8 & 35.4 & 21.6 & 20.7 & 58 & 53 \\
\hline Total & $1,759.7$ & $1,759.1$ & 70.9 & 59.3 & $-^{\mathrm{a}}$ & $--^{\mathrm{a}}$ & $-^{\mathrm{a}}$ & $-^{\mathrm{a}}$ & $--^{\mathrm{a}}$ & $--^{\mathrm{a}}$ \\
\hline Mean & 146.64 & 146.59 & 5.91 & 4.94 & 33.52 & 34.39 & 23.16 & 22.97 & 71.33 & 70.50 \\
\hline$t_{\mathrm{df}=11 ; a=0.05}$ & \multicolumn{2}{|c|}{ NS } & \multicolumn{2}{|c|}{ NS } & \multicolumn{2}{|c|}{ NS } & \multicolumn{2}{|c|}{ NS } & \multicolumn{2}{|c|}{ NS } \\
\hline
\end{tabular}

$\mathrm{NS}=$ : paired student's $t$-test of the mean weather condition for the 2 years is not significant at $95 \%$ confidence level.

${ }^{\mathrm{a}}$ :cCalculation of cumulative value (i.e., total) not applicable.

and plant height $(\mathrm{cm})$; at full maturity and harvest (by uprooting each plant in the sample plot), the following data were obtained: number of leaves per plant, leaf area $\left(\mathrm{cm}^{2}\right)$, number of tillers per plant, number of rhizome fingers per plant, rhizome length $(\mathrm{cm})$, and rhizome yield (t/ha). Data obtained were checked for homogeneity of variance and normality using Levene and Shapiro-Wilk tests, respectively, before being subjected to a twoway analysis of variance (ANOVA) using GenStat ${ }^{\circledR}$ for Windows ${ }^{\circledR}$ version 8.1 (VSN International Ltd, Hemel Hempstead, UK), and significant means were separated using Duncan Multiple Range 
Table 4. Mean sprouting percentages (\%) of 17 ginger lines in Calabar, Ikom, and Ogoja at 4WAP (2016 and 2017 early season plantings).

\begin{tabular}{|c|c|c|c|c|c|c|c|c|c|c|c|c|}
\hline \multirow{4}{*}{ Ginger line } & \multirow{4}{*}{ Genotype* } & \multirow{2}{*}{\multicolumn{2}{|c|}{$\begin{array}{c}\text { Genotype } \times \text { year }^{\mathrm{NS}} \\
\text { Year }^{\mathrm{NS}}\end{array}$}} & \multirow{2}{*}{\multicolumn{3}{|c|}{$\begin{array}{c}\text { Genotype } \times \text { location }^{\mathrm{NS}} \\
\text { Location }^{\mathrm{NS}}\end{array}$}} & \multirow{2}{*}{\multicolumn{6}{|c|}{$\begin{array}{c}\text { Genotype } \times \text { year } \times \text { location }^{\mathrm{NS}} \\
\text { Year } \times \text { location }^{\mathrm{NS}}\end{array}$}} \\
\hline & & & & & & & & & & & & \\
\hline & & \multirow{2}{*}{2016} & \multirow{2}{*}{2017} & \multirow{2}{*}{ Calabar } & \multirow{2}{*}{ Ikom } & \multirow{2}{*}{ Ogoja } & \multicolumn{3}{|c|}{2016} & \multicolumn{3}{|c|}{2017} \\
\hline & & & & & & & Calabar & Ikom & Ogoja & Calabar & Ikom & Ogoja \\
\hline UG1 & $62.89^{c}$ & $62.89^{\mathrm{a}}$ & $62.89^{\mathrm{a}}$ & $56.50^{\mathrm{a}}$ & $63.00^{\mathrm{a}}$ & $69.17^{\mathrm{a}}$ & $62.67^{\mathrm{a}}$ & $63.00^{\mathrm{a}}$ & $63.00^{\mathrm{a}}$ & $50.33^{\mathrm{a}}$ & $63.00^{\mathrm{a}}$ & $75.33^{\mathrm{a}}$ \\
\hline UG1-11-07 & $81.50^{\mathrm{a}}$ & $83.56^{\mathrm{a}}$ & $79.44^{\mathrm{a}}$ & $73.17^{\mathrm{a}}$ & $85.67^{\mathrm{a}}$ & $85.67^{\mathrm{a}}$ & $79.33^{\mathrm{a}}$ & $87.67^{\mathrm{a}}$ & $83.67^{\mathrm{a}}$ & $67.00^{\mathrm{a}}$ & $83.67^{\mathrm{a}}$ & $87.67^{\mathrm{a}}$ \\
\hline UG1-13-02 & $66.89^{\mathrm{bc}}$ & $62.78^{\mathrm{a}}$ & $71.00^{\mathrm{a}}$ & $62.83^{\mathrm{a}}$ & $66.83^{\mathrm{a}}$ & $71.00^{\mathrm{a}}$ & $54.67^{\mathrm{a}}$ & $62.67^{\mathrm{a}}$ & $71.00^{\mathrm{a}}$ & $71.00^{\mathrm{a}}$ & $71.00^{\mathrm{a}}$ & $71.00^{\mathrm{a}}$ \\
\hline UG1-2-35 & $63.44^{c}$ & $62.78^{\mathrm{a}}$ & $64.11^{\mathrm{a}}$ & $62.83^{\mathrm{a}}$ & $60.83^{\mathrm{a}}$ & $66.67^{\mathrm{a}}$ & $58.67^{\mathrm{a}}$ & $63.00^{\mathrm{a}}$ & $66.67^{\mathrm{a}}$ & $67.00^{\mathrm{a}}$ & $58.67^{\mathrm{a}}$ & $66.67^{\mathrm{a}}$ \\
\hline UG1-5-04 & $71.06^{\mathrm{abc}}$ & $68.22^{\mathrm{a}}$ & $73.89^{\mathrm{a}}$ & $73.17^{\mathrm{a}}$ & $65.00^{\mathrm{a}}$ & $75.00^{\mathrm{a}}$ & $62.67^{\mathrm{a}}$ & $67.00^{\mathrm{a}}$ & $75.00^{\mathrm{a}}$ & $83.67^{\mathrm{a}}$ & $63.00^{\mathrm{a}}$ & $75.00^{\mathrm{a}}$ \\
\hline UG1-5-18 & $64.11^{\mathrm{c}}$ & $60.00^{\mathrm{a}}$ & $68.22^{\mathrm{a}}$ & $52.17^{\mathrm{a}}$ & $71.17^{\mathrm{a}}$ & $69.00^{\mathrm{a}}$ & $33.33^{\mathrm{a}}$ & $79.67^{\mathrm{a}}$ & $67.00^{\mathrm{a}}$ & $71.00^{\mathrm{a}}$ & $62.67^{\mathrm{a}}$ & $71.00 \mathrm{v}$ \\
\hline UG1-5-22 & $73.11^{\mathrm{abc}}$ & $76.56^{\mathrm{a}}$ & $69.67^{\mathrm{a}}$ & $69.00^{\mathrm{a}}$ & $71.00^{\mathrm{a}}$ & $79.33^{\mathrm{a}}$ & $79.33^{\mathrm{a}}$ & $71.00^{\mathrm{a}}$ & $79.33^{\mathrm{a}}$ & $58.67^{\mathrm{a}}$ & $71.00^{\mathrm{a}}$ & $79.33^{\mathrm{a}}$ \\
\hline UG1-5-31 & $67.61^{\mathrm{bc}}$ & $64.22^{\mathrm{a}}$ & $71.00^{\mathrm{a}}$ & $67.00^{\mathrm{a}}$ & $73.17^{\mathrm{a}}$ & $62.67^{\mathrm{a}}$ & $50.33^{\mathrm{a}}$ & $79.67^{\mathrm{a}}$ & $62.67^{\mathrm{a}}$ & $83.67^{\mathrm{a}}$ & $66.67^{\mathrm{a}}$ & $62.67^{\mathrm{a}}$ \\
\hline UG1-5-35 & $64.72^{c}$ & $63.89^{\mathrm{a}}$ & $65.56^{\mathrm{a}}$ & $52.50^{\mathrm{a}}$ & $73.00^{\mathrm{a}}$ & $68.67^{\mathrm{a}}$ & $42.00^{\mathrm{a}}$ & $79.33^{\mathrm{a}}$ & $70.33^{\mathrm{a}}$ & $63.00^{\mathrm{a}}$ & $66.67^{\mathrm{a}}$ & $67.00^{\mathrm{a}}$ \\
\hline UG1-5-38 & $61.33^{\mathrm{c}}$ & $59.89^{\mathrm{a}}$ & $62.78^{\mathrm{a}}$ & $60.67^{\mathrm{a}}$ & $60.67^{\mathrm{a}}$ & $62.67^{\mathrm{a}}$ & $50.33^{\mathrm{a}}$ & $66.67^{\mathrm{a}}$ & $62.67^{\mathrm{a}}$ & $71.00^{\mathrm{a}}$ & $54.67^{\mathrm{a}}$ & $62.67^{\mathrm{a}}$ \\
\hline UG1-5-48 & $80.11^{\mathrm{ab}}$ & $74.44^{\mathrm{a}}$ & $80.78^{\mathrm{a}}$ & $75.33^{\mathrm{a}}$ & $69.00^{\mathrm{a}}$ & $96.00^{\mathrm{a}}$ & $67.00^{\mathrm{a}}$ & $75.33^{\mathrm{a}}$ & $96.00^{\mathrm{a}}$ & $83.67^{\mathrm{a}}$ & $62.67 \mathrm{v}$ & $96.00^{\mathrm{a}}$ \\
\hline UG1-5-49 & $66.83^{\mathrm{bc}}$ & $62.67^{\mathrm{a}}$ & $71.00^{\mathrm{a}}$ & $62.50^{\mathrm{a}}$ & $60.67^{\mathrm{a}}$ & $77.33^{\mathrm{a}}$ & $41.67^{\mathrm{a}}$ & $67.00^{\mathrm{a}}$ & $79.33^{\mathrm{a}}$ & $83.33^{\mathrm{a}}$ & $54.33^{\mathrm{a}}$ & $75.00^{\mathrm{a}}$ \\
\hline UG1-5-52 & $66.22^{\mathrm{c}}$ & $64.11^{\mathrm{a}}$ & $68.33^{\mathrm{a}}$ & $71.17^{\mathrm{a}}$ & $56.50^{\mathrm{a}}$ & $71.00^{\mathrm{a}}$ & $58.67^{\mathrm{a}}$ & $62.67^{\mathrm{a}}$ & $71.00^{\mathrm{a}}$ & $83.67^{\mathrm{a}}$ & $50.33^{\mathrm{a}}$ & $71.00^{\mathrm{a}}$ \\
\hline UG1-7-24 & $73.11^{\mathrm{abc}}$ & $68.22^{\mathrm{a}}$ & $78.00^{\mathrm{a}}$ & $66.83^{\mathrm{a}}$ & $75.17^{\mathrm{a}}$ & $77.33^{\mathrm{a}}$ & $46.00^{\mathrm{a}}$ & $79.33^{\mathrm{a}}$ & $79.33^{\mathrm{a}}$ & $87.67^{\mathrm{a}}$ & $71.00^{\mathrm{a}}$ & $75.33^{\mathrm{a}}$ \\
\hline UG2 & $66.97^{\mathrm{bc}}$ & $64.17^{\mathrm{a}}$ & $69.78^{\mathrm{a}}$ & $68.92^{\mathrm{a}}$ & $69.17^{\mathrm{a}}$ & $62.83^{\mathrm{a}}$ & $62.50^{\mathrm{a}}$ & $71.33^{\mathrm{a}}$ & $58.67^{\mathrm{a}}$ & $75.33^{\mathrm{a}}$ & $67.00^{\mathrm{a}}$ & $67.00^{\mathrm{a}}$ \\
\hline UG2-11-03 & $69.72^{\mathrm{abc}}$ & $68.22^{\mathrm{a}}$ & $71.22^{\mathrm{a}}$ & $58.67^{\mathrm{a}}$ & $77.00^{\mathrm{a}}$ & $73.33^{\mathrm{a}}$ & $46.00^{\mathrm{a}}$ & $83.33^{\mathrm{a}}$ & $75.33^{\mathrm{a}}$ & $71.33^{\mathrm{a}}$ & $71.00^{\mathrm{a}}$ & $71.33^{\mathrm{a}}$ \\
\hline UG2-9-01 & $74.44^{\mathrm{abc}}$ & $73.67^{\mathrm{a}}$ & $75.22^{\mathrm{a}}$ & $58.50^{\mathrm{a}}$ & $83.50^{\mathrm{a}}$ & $81.33^{\mathrm{a}}$ & $54.33^{\mathrm{a}}$ & $83.33^{\mathrm{a}}$ & $83.33^{\mathrm{a}}$ & $62.67^{\mathrm{a}}$ & $83.67^{\mathrm{a}}$ & $79.33^{\mathrm{a}}$ \\
\hline Mean $^{\mathrm{a}}$ & 69.06 & 67.37 & 70.76 & 64.22 & 69.5 & 73.46 & 55.55 & 73.06 & 73.20 & 72.53 & 65.94 & 73.75 \\
\hline SEM & 1.15 & 1.83 & 1.39 & 2.45 & 1.61 & 1.54 & 3.06 & 2.04 & 2.34 & 2.57 & 2.21 & 2.08 \\
\hline CV (\%) & 16.89 & 19.40 & 13.99 & 22.21 & 13.48 & 12.23 & 22.55 & 11.53 & 13.18 & 14.58 & 13.83 & 11.62 \\
\hline
\end{tabular}

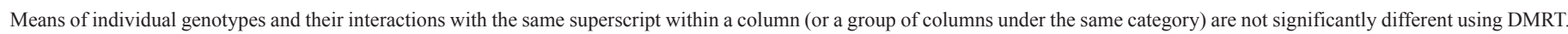
$\mathrm{NS}=$ : not significant.

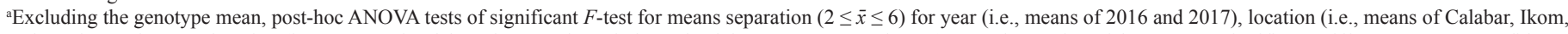

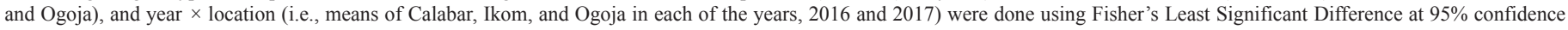
level $\left(\operatorname{LSD}_{0.05}\right)$.

*Significant at $p<0.05$.

$* *$ Significant at $p<0.01$.

Test (DMRT) at 5\% level of significance [12]. Square-root $(\sqrt{ } x+$ 1) transformation was used for count data while percentages were $\log _{10}$ transformed. Interpreted results of the transformed data were back-transformed for presentation.

\section{RESULTS AND DISCUSSION}

\subsection{Sprouting Percentage at 4 Weeks after Planting (4WAP)}

Combined ANOVA showed highly significant differences in the sprouting percentages (4WAP) among the genotypes $(\mathrm{p}<0.01)$ (Table 4). Years and locations showed no significant differences in sprouting percentage $(p>0.05)$. Year by genotype interaction, location by genotype interaction, and year by genotype by location interactions were also not significant. Although the difference was not significant $(p>0.05)$, the mean sprouting percentage in $2017(70.76 \%)$ was higher than that of 2016 (67.37\%). Across the three locations, Ogoja had the highest mean sprouting percentage (73.47\%), followed by Ikom (69.50\%), while Calabar (64.22\%) had the lowest.

\subsection{Establishment Count at 8 Weeks after Planting (8WAP)}

The establishment counts of 17 ginger lines at $8 \mathrm{WAP}$ across three locations in 2 years are presented in Table 5. Combined ANOVA indicated that there was no significant difference $(p>0.05)$ in the establishment count of the ginger lines across genotypes, years, locations, and genotype by environment interactions.

\subsubsection{Plant height}

As presented in Table 6, ANOVA showed that plant height was significantly $(p<0.01)$ different across genotypes, location, and year. Year by genotype interaction and year by location by genotype interaction were not significant $(p>0.05)$. However, location by genotype interaction was very highly significant $(p<0.001)$. Among the genotypes, UG2-11-03 and UG1-7-24 produced the tallest plants. Across the three locations, the tallest plants were observed in Ogoja followed by those planted in Ikom then Calabar. The ginger lines planted in $2017(57.83 \mathrm{~cm})$ were significantly taller than those planted in $2016(53.47 \mathrm{~cm})$. 
Table 5. Establishment count (\%) of 17 ginger lines in Calabar, Ikom, and Ogoja at 8WAP (2016 and 2017 early season plantings).

\begin{tabular}{|c|c|c|c|c|c|c|c|c|c|c|c|c|}
\hline \multirow{3}{*}{ Ginger line } & \multirow{3}{*}{ Genotype $^{\mathrm{NS}}$} & \multirow{2}{*}{\multicolumn{2}{|c|}{$\begin{array}{c}\text { Genotype } \times \text { year }^{\mathrm{NS}} \\
\text { Year }^{\mathrm{NS}}\end{array}$}} & \multirow{2}{*}{\multicolumn{3}{|c|}{$\begin{array}{c}\text { Genotype } \times \text { location }^{\mathrm{NS}} \\
\text { Location }^{\mathrm{NS}}\end{array}$}} & \multirow{2}{*}{\multicolumn{6}{|c|}{$\begin{array}{c}\text { Genotype } \times \text { year } \times \text { location }^{\mathrm{NS}} \\
\text { Year } \times \text { location }^{\mathrm{NS}}\end{array}$}} \\
\hline & & & & & & & & & & & & \\
\hline & & 2016 & 2017 & Calabar & Ikom & Ogoja & \multicolumn{3}{|c|}{2016} & \multicolumn{3}{|c|}{2017} \\
\hline UG1 & $76.16^{\mathrm{a}}$ & $78.00^{\mathrm{a}}$ & $75.22^{\mathrm{a}}$ & $69.00^{\mathrm{a}}$ & $83.50^{\mathrm{a}}$ & $77.33^{\mathrm{a}}$ & $71.00^{\mathrm{a}}$ & $87.67^{\mathrm{a}}$ & $75.33^{\mathrm{a}}$ & $67.00^{\mathrm{a}}$ & $79.33^{\mathrm{a}}$ & $79.33^{\mathrm{a}}$ \\
\hline UG1-11-07 & $88.39^{a}$ & $87.78^{\mathrm{a}}$ & $89.00^{\mathrm{a}}$ & $93.83^{\mathrm{a}}$ & $83.67^{\mathrm{a}}$ & $87.67^{\mathrm{a}}$ & $87.67^{\mathrm{a}}$ & $92.00^{\mathrm{a}}$ & $83.67^{\mathrm{a}}$ & $100.00^{\mathrm{a}}$ & $75.33^{\mathrm{a}}$ & $91.67^{\mathrm{a}}$ \\
\hline UG1-13-02 & $77.33^{\mathrm{a}}$ & $78.11^{\mathrm{a}}$ & $76.56^{\mathrm{a}}$ & $73.17^{\mathrm{a}}$ & $77.33^{\mathrm{a}}$ & $81.50^{\mathrm{a}}$ & $67.00^{\mathrm{a}}$ & $83.67^{\mathrm{a}}$ & $83.67^{\mathrm{a}}$ & $79.33^{\mathrm{a}}$ & $71.00^{\mathrm{a}}$ & $79.33^{\mathrm{a}}$ \\
\hline UG1-5-04 & $80.33^{\mathrm{a}}$ & $83.67^{\mathrm{a}}$ & $77.00^{\mathrm{a}}$ & $79.50^{\mathrm{a}}$ & $80.00^{\mathrm{a}}$ & $81.50^{\mathrm{a}}$ & $75.33^{\mathrm{a}}$ & $88.00^{\mathrm{a}}$ & $87.67^{\mathrm{a}}$ & $83.67^{a}$ & $72.00^{\mathrm{a}}$ & $75.33^{\mathrm{a}}$ \\
\hline UG1-5-18 & $73.06^{\mathrm{a}}$ & $70.89^{a}$ & $75.22^{\mathrm{a}}$ & $64.67^{\mathrm{a}}$ & $79.33^{\mathrm{a}}$ & $75.17^{\mathrm{a}}$ & $50.00^{\mathrm{a}}$ & $83.33^{\mathrm{a}}$ & $79.33^{\mathrm{a}}$ & $73.33^{\mathrm{a}}$ & $75.33^{\mathrm{a}}$ & $71.00^{\mathrm{a}}$ \\
\hline UG1-5-22 & $82.11^{\mathrm{a}}$ & $86.22^{\mathrm{a}}$ & $78.00^{\mathrm{a}}$ & $91.83^{\mathrm{a}}$ & $66.83^{\mathrm{a}}$ & $87.67^{\mathrm{a}}$ & $100.00^{\mathrm{a}}$ & $75.00^{\mathrm{a}}$ & $83.67^{\mathrm{a}}$ & $83.67^{\mathrm{a}}$ & $58.67^{\mathrm{a}}$ & $91.67^{\mathrm{a}}$ \\
\hline UG1-5-31 & $77.28^{\mathrm{a}}$ & $78.00^{\mathrm{a}}$ & $76.56^{\mathrm{a}}$ & $77.33^{\mathrm{a}}$ & $81.33^{\mathrm{a}}$ & $73.17^{\mathrm{a}}$ & $75.33^{a}$ & $87.67^{\mathrm{a}}$ & $71.00^{\mathrm{a}}$ & $79.33^{\mathrm{a}}$ & $75.00^{\mathrm{a}}$ & $75.33^{\mathrm{a}}$ \\
\hline UG1-5-35 & $74.56^{\mathrm{a}}$ & $80.78^{\mathrm{a}}$ & $68.33^{\mathrm{a}}$ & $89.83^{\mathrm{a}}$ & $69.33^{\mathrm{a}}$ & $64.83^{\mathrm{a}}$ & $87.67^{\mathrm{a}}$ & $83.67^{\mathrm{a}}$ & $71.00^{\mathrm{a}}$ & $92.00^{\mathrm{a}}$ & $54.33^{\mathrm{a}}$ & $58.67^{\mathrm{a}}$ \\
\hline UG1-5-52 & $75.22^{\mathrm{a}}$ & $76.56^{\mathrm{a}}$ & $73.89^{\mathrm{a}}$ & $81.50^{\mathrm{a}}$ & $64.83^{\mathrm{a}}$ & $79.33^{\mathrm{a}}$ & $71.00^{\mathrm{a}}$ & $71.00^{\mathrm{a}}$ & $87.67^{\mathrm{a}}$ & $92.00^{\mathrm{a}}$ & $58.67^{\mathrm{a}}$ & $71.00^{\mathrm{a}}$ \\
\hline UG1-7-24 & $82.17^{\mathrm{a}}$ & $80.78^{\mathrm{a}}$ & $83.56^{\mathrm{a}}$ & $79.50^{\mathrm{a}}$ & $81.33^{\mathrm{a}}$ & $85.67^{\mathrm{a}}$ & $67.00^{\mathrm{a}}$ & $83.33^{\mathrm{a}}$ & $92.00^{\mathrm{a}}$ & $92.00^{\mathrm{a}}$ & $79.33^{\mathrm{a}}$ & $79.33^{\mathrm{a}}$ \\
\hline UG2 & $78.06^{\mathrm{a}}$ & $78.00^{\mathrm{a}}$ & $78.11^{\mathrm{a}}$ & $87.60^{\mathrm{a}}$ & $73.33^{\mathrm{a}}$ & $73.17^{\mathrm{a}}$ & $91.67^{\mathrm{a}}$ & $71.33^{\mathrm{a}}$ & $71.00^{\mathrm{a}}$ & $83.67^{\mathrm{a}}$ & $75.33^{\mathrm{a}}$ & $75.33^{\mathrm{a}}$ \\
\hline UG2-11-03 & $84.22^{\mathrm{a}}$ & $87.67^{\mathrm{a}}$ & $80.78^{\mathrm{a}}$ & $73.17^{\mathrm{a}}$ & $87.67^{\mathrm{a}}$ & $91.83^{\mathrm{a}}$ & $71.00^{\mathrm{a}}$ & $96.00^{\mathrm{a}}$ & $96.00^{\mathrm{a}}$ & $75.33^{\mathrm{a}}$ & $79.33^{\mathrm{a}}$ & $87.67^{\mathrm{a}}$ \\
\hline UG2-9-01 & $80.72^{\mathrm{a}}$ & $79.33^{\mathrm{a}}$ & $82.11^{\mathrm{a}}$ & $75.17^{\mathrm{a}}$ & $85.67^{\mathrm{a}}$ & $81.33^{\mathrm{a}}$ & $71.00^{\mathrm{a}}$ & $83.67^{\mathrm{a}}$ & $83.33^{\mathrm{a}}$ & $79.33^{\mathrm{a}}$ & $87.67^{\mathrm{a}}$ & $79.33^{\mathrm{a}}$ \\
\hline Mean $^{a}$ & 79.1 & 80.93 & 77.38 & 79.71 & 77.71 & 79.87 & 76.92 & 83.80 & 82.08 & 82.84 & 71.63 & 77.67 \\
\hline SEM & 0.97 & 1.36 & 1.34 & 1.78 & 1.74 & 1.51 & 2.88 & 1.84 & 2.02 & 1.97 & 2.13 & 2.18 \\
\hline CV (\%) & 12.35 & 12.00 & 12.39 & 13.04 & 13.08 & 11.05 & 15.42 & 9.03 & 10.16 & 9.82 & 12.26 & 11.57 \\
\hline
\end{tabular}

Means of individual genotypes and their interactions with the same superscript within a column (or a group of columns under the same category) are not significantly different using DMRT.

NS =: not significant at $5 \%$ probability level.

${ }^{a}$ Excluding the genotype mean, post-hoc ANOVA tests of significant $F$-test for means separation $(2 \leq \bar{x} \leq 6)$ for year (i.e., means of 2016 and 2017$)$, location (i.e., means of Calabar, Ikom, and Ogoja), and year $\times$ location (i.e., means of Calabar, Ikom, and Ogoja in each of the years, 2016 and 2017) were done using Fisher's Least Significant Difference at 95\% confidence level $\left(\mathrm{LSD}_{0.05}\right)$.

\subsubsection{Number of leaves per plant}

Combined ANOVA (Table 7) showed that genotype $(p<0.01)$ and location $(p<0.05)$, year by genotype interaction $(p<0.05)$, and location by genotype interaction $(p<0.01)$ significantly affected the number of leaves per plant of the ginger lines. Year and year by location by genotype interaction were not significant $(p>0.05)$. The mutant line, UG1-13-02, produced the highest number of leaves per plant and was similar to check UG1 but significantly different $(p<0.05)$ from check UG2. Overall, ginger lines planted in Ikom had the highest number of leaves per plant followed by Calabar while Ogoja had plants with the fewest number of leaves.

\subsubsection{Leaf area}

Highly significant $(p<0.01)$ differences in leaf area were observed across genotypes, locations, years, and location by genotype interaction (Table 8). Year by genotype interaction and year by location by genotype interaction were not significant ( $p$ $>0.05$ ). The leave sizes of each of the checks (UG1 and UG2) were larger than those of their mutant derivatives. Ginger lines planted in Ogoja produced significantly larger leaves than those planted in Ikom and Calabar. The leaves of ginger planted in 2017 $\left(34.37 \mathrm{~cm}^{2}\right)$ were significantly larger than those planted in 2016 $\left(31.53 \mathrm{~cm}^{2}\right)$. In Ikom, the largest leaves were produced by UG1 and UG2, while in Calabar, they were below the location average. In Ogoja, UG2 had the highest mean leaf area, while UG1-2-35 plants produced significantly smaller mean leaf area.

\subsubsection{Number of tillers per plant}

The number of tillers per plant was significantly $(p<0.01)$ affected by genotype and location by genotype interaction (Table 9 ). Location, year by genotype interaction, and genotype by year by location interaction did not significantly $(p>0.05)$ affect the number of tillers per plant. UG2-11-03 produced the highest number of tillers per plant and was not significantly different from the checks. UG1-5-04 produced the least number of tillers per plant and was not significantly different $(p>0.05)$ from all the UG1-5 mutants. Ginger lines planted in 2016 had a significantly ( $p$ $<0.05)$ higher number of tillers (16.40) than those of 2017 (10.20). The number of tillers had an inconsistent trend across locations. 

2021;9(05):110-123

Table 6. Mean plant height $(\mathrm{cm})$ of 17 ginger lines in Calabar, Ikom, and Ogoja (2016 and 2017 early season plantings).

\begin{tabular}{|c|c|c|c|c|c|c|c|c|c|c|c|c|}
\hline \multirow{4}{*}{ Ginger line } & \multirow{4}{*}{ Genotype* } & \multirow{2}{*}{\multicolumn{2}{|c|}{$\begin{array}{c}\text { Genotype } \times \text { year }^{\text {NS }} \\
\text { Year* }\end{array}$}} & \multirow{2}{*}{\multicolumn{3}{|c|}{$\begin{array}{l}\text { Genotype } \times \text { location* } \\
\text { Location* }\end{array}$}} & \multirow{2}{*}{\multicolumn{6}{|c|}{$\begin{array}{c}\text { Genotype } \times \text { year } \times \text { location }^{\mathrm{NS}} \\
\text { Year } \times \text { location }^{\mathrm{NS}}\end{array}$}} \\
\hline & & & & & & & & & & & & \\
\hline & & \multirow{2}{*}{2016} & \multirow{2}{*}{2017} & \multirow{2}{*}{ Calabar } & \multirow{2}{*}{ Ikom } & \multirow{2}{*}{ Ogoja } & \multicolumn{3}{|c|}{2016} & \multicolumn{3}{|c|}{2017} \\
\hline & & & & & & & Calabar & Ikom & Ogoja & Calabar & Ikom & Ogoja \\
\hline UG1 & $56.76^{\text {bcd }}$ & $53.96^{\mathrm{a}}$ & $59.56^{\mathrm{a}}$ & $34.93^{\operatorname{lm}}$ & $65.00^{\mathrm{b}-\mathrm{g}}$ & $70.33^{\mathrm{a}-\mathrm{d}}$ & $28.87^{\mathrm{a}}$ & $63.33^{\mathrm{a}}$ & $69.67^{\mathrm{a}}$ & $41.00^{\mathrm{a}}$ & $66.67^{\mathrm{a}}$ & $71.00^{\mathrm{a}}$ \\
\hline UG1-11-07 & $52.51^{\mathrm{c}-\mathrm{f}}$ & $53.36^{\mathrm{a}}$ & $51.67^{\mathrm{a}}$ & $37.93^{\mathrm{klm}}$ & $55.27^{\mathrm{e}-\mathrm{j}}$ & $64.33^{\mathrm{c}-\mathrm{g}}$ & $32.53^{\mathrm{a}}$ & $56.53^{\mathrm{a}}$ & $71.00^{\mathrm{a}}$ & $43.33^{\mathrm{a}}$ & $54.00^{\mathrm{a}}$ & $57.67^{\mathrm{a}}$ \\
\hline UG1-13-02 & $60.03^{b}$ & $57.17^{\mathrm{a}}$ & $62.89^{\mathrm{a}}$ & $43.83^{\mathrm{jkl}}$ & $63.83^{\mathrm{c}-\mathrm{g}}$ & $72.42^{\mathrm{abc}}$ & $31.67^{\mathrm{a}}$ & $65.67^{\mathrm{a}}$ & $74.17^{\mathrm{a}}$ & $56.00^{\mathrm{a}}$ & $62.00^{\mathrm{a}}$ & $70.67^{\mathrm{a}}$ \\
\hline UG1-2-35 & $54.94^{\text {be }}$ & $54.78^{\mathrm{a}}$ & $55.11^{\mathrm{a}}$ & $36.67^{\mathrm{Im}}$ & $62.67^{\mathrm{c}-\mathrm{g}}$ & $65.50^{\mathrm{b}-\mathrm{g}}$ & $32.00^{\mathrm{a}}$ & $63.00^{\mathrm{a}}$ & $69.33^{\mathrm{a}}$ & $41.33^{\mathrm{a}}$ & $62.33^{\mathrm{a}}$ & $61.67^{\mathrm{a}}$ \\
\hline UG1-5-04 & $53.39^{\mathrm{b}-\mathrm{f}}$ & $52.00^{\mathrm{a}}$ & $54.78^{\mathrm{a}}$ & $36.00^{\operatorname{lm}}$ & $54.33^{\mathrm{f}-\mathrm{j}}$ & $69.83^{\mathrm{a}-\mathrm{d}}$ & $29.67^{a}$ & $55.00^{\mathrm{a}}$ & $71.33^{\mathrm{a}}$ & $42.33^{\mathrm{a}}$ & $53.67^{\mathrm{a}}$ & $68.33^{\mathrm{a}}$ \\
\hline UG1-5-18 & $46.74^{\mathrm{f}}$ & $42.71^{\mathrm{a}}$ & $50.78^{\mathrm{a}}$ & $30.07^{\mathrm{m}}$ & $43.33^{\mathrm{jkl}}$ & $66.83^{\mathrm{b}-\mathrm{f}}$ & $19.47^{\mathrm{a}}$ & $40.67^{\mathrm{a}}$ & $68.00^{\mathrm{a}}$ & $40.67^{\mathrm{a}}$ & $46.00^{\mathrm{a}}$ & $65.67^{\mathrm{a}}$ \\
\hline UG1-5-22 & $53.66^{\mathrm{b}-\mathrm{f}}$ & $52.87^{\mathrm{a}}$ & $54.44^{\mathrm{a}}$ & $39.27^{\mathrm{klm}}$ & $50.12^{\mathrm{h}-\mathrm{k}}$ & $71.58^{\mathrm{abc}}$ & $33.20^{\mathrm{a}}$ & $52.23^{\mathrm{a}}$ & $73.17^{\mathrm{a}}$ & $45.33^{\mathrm{a}}$ & $48.00^{\mathrm{a}}$ & $70.00^{\mathrm{a}}$ \\
\hline UG1-5-31 & $52.53^{\mathrm{c}-\mathrm{f}}$ & $51.39^{\mathrm{a}}$ & $53.67^{\mathrm{a}}$ & $36.37^{\mathrm{lm}}$ & $57.72^{\mathrm{d}-\mathrm{i}}$ & $63.50^{c-g}$ & $32.07^{\mathrm{a}}$ & $57.43^{\mathrm{a}}$ & $64.67^{\mathrm{a}}$ & $40.67^{\mathrm{a}}$ & $58.00^{\mathrm{a}}$ & $62.33^{\mathrm{a}}$ \\
\hline UG1-5-35 & $53.31^{\mathrm{b}-\mathrm{f}}$ & $51.18^{\mathrm{a}}$ & $55.44^{\mathrm{a}}$ & $43.17^{\mathrm{jkl}}$ & $45.17^{\mathrm{i}-1}$ & $71.60^{\mathrm{abc}}$ & $36.00^{\mathrm{a}}$ & $44.00^{\mathrm{a}}$ & $73.53^{\mathrm{a}}$ & $50.33^{\mathrm{a}}$ & $46.33^{\mathrm{a}}$ & $69.67^{\mathrm{a}}$ \\
\hline UG1-5-38 & $50.75^{\mathrm{def}}$ & $49.61^{a}$ & $51.89^{\mathrm{a}}$ & $36.20^{\operatorname{lm}}$ & $53.72^{\mathrm{g}-\mathrm{j}}$ & $62.33^{\mathrm{c}-\mathrm{h}}$ & $31.73^{\mathrm{a}}$ & $53.10^{\mathrm{a}}$ & $64.00^{\mathrm{a}}$ & $40.67^{a}$ & $54.33^{\mathrm{a}}$ & $60.67^{\mathrm{a}}$ \\
\hline UG1-5-48 & $56.52^{\mathrm{bcd}}$ & $54.04^{\mathrm{a}}$ & $59.00^{\mathrm{a}}$ & $45.80^{\mathrm{ijk} \mathrm{l}}$ & $50.27^{\mathrm{h}-\mathrm{k}}$ & $73.50^{\mathrm{abc}}$ & $37.60^{\mathrm{a}}$ & $49.20^{\mathrm{a}}$ & $75.33^{\mathrm{a}}$ & $54.00^{\mathrm{a}}$ & $51.33^{\mathrm{a}}$ & $71.67^{\mathrm{a}}$ \\
\hline UG1-5-49 & $53.52^{\mathrm{b}-\mathrm{f}}$ & $52.38^{\mathrm{a}}$ & $54.67^{\mathrm{a}}$ & $37.57^{\mathrm{klm}}$ & $56.00^{e-j}$ & $67.00^{\mathrm{b}-\mathrm{f}}$ & $29.47^{\mathrm{a}}$ & $57.67^{a}$ & $70.00^{\mathrm{a}}$ & $45.67^{\mathrm{a}}$ & $54.33^{\mathrm{a}}$ & $64.00^{\mathrm{a}}$ \\
\hline UG1-5-52 & $49.08^{\mathrm{ef}}$ & $46.49^{\mathrm{a}}$ & $51.67^{\mathrm{a}}$ & $40.23^{\mathrm{klm}}$ & $43.33^{\mathrm{jkl}}$ & $63.67^{\mathrm{c}-\mathrm{g}}$ & $30.80^{\mathrm{a}}$ & $44.00^{\mathrm{a}}$ & $64.67^{\mathrm{a}}$ & $49.67^{\mathrm{a}}$ & $42.67^{\mathrm{a}}$ & $62.67^{\mathrm{a}}$ \\
\hline UG1-7-24 & $67.06^{\mathrm{a}}$ & $64.89^{\mathrm{a}}$ & $69.22^{\mathrm{a}}$ & $44.97^{\mathrm{jkl}}$ & $74.70^{\mathrm{abc}}$ & $81.50^{\mathrm{a}}$ & $33.27^{\mathrm{a}}$ & $77.40^{\mathrm{a}}$ & $84.00^{\mathrm{a}}$ & $56.67^{\mathrm{a}}$ & $72.00^{\mathrm{a}}$ & $79.00^{\mathrm{a}}$ \\
\hline UG2 & $58.67^{\mathrm{bc}}$ & $53.68^{\mathrm{a}}$ & $63.67^{\mathrm{a}}$ & $39.27^{\mathrm{klm}}$ & $69.17^{\mathrm{a}-\mathrm{d}}$ & $67.58^{\mathrm{b}-\mathrm{e}}$ & $27.87^{\mathrm{a}}$ & $68.00^{\mathrm{a}}$ & $65.17^{\mathrm{a}}$ & $50.67^{\mathrm{a}}$ & $70.33^{\mathrm{a}}$ & $70.00^{\mathrm{a}}$ \\
\hline UG2-11-03 & $67.45^{\mathrm{a}}$ & $63.12^{\mathrm{a}}$ & $71.78^{\mathrm{a}}$ & $46.80^{\mathrm{ijkl}}$ & $77.88^{\mathrm{ab}}$ & $77.67^{\mathrm{ab}}$ & $34.27^{\mathrm{a}}$ & $74.43^{\mathrm{a}}$ & $80.67^{\mathrm{a}}$ & $59.33^{\mathrm{a}}$ & $81.33^{\mathrm{a}}$ & $74.67^{\mathrm{a}}$ \\
\hline UG2-9-01 & $59.16^{\mathrm{bc}}$ & $55.43^{\mathrm{a}}$ & $62.89^{\mathrm{a}}$ & $39.90^{\mathrm{klm}}$ & $64.25^{\mathrm{c}-\mathrm{g}}$ & $73.33^{\mathrm{abc}}$ & $29.80^{\mathrm{a}}$ & $63.17^{\mathrm{a}}$ & $73.33^{\mathrm{a}}$ & $50.00^{\mathrm{a}}$ & $65.33^{\mathrm{a}}$ & $73.00^{\mathrm{a}}$ \\
\hline Mean $^{a}$ & 55.65 & 53.47 & 57.82 & 39.35 & 58.04 & 69.55 & 31.19 & 57.93 & 71.30 & 47.51 & 58.16 & 67.82 \\
\hline SEM & 1.51 & 2.55 & 1.59 & 1.68 & 1.77 & 0.99 & 0.95 & 2.52 & 1.33 & 1.53 & 2.56 & 1.37 \\
\hline CV (\%) & 27.44 & 34.11 & 19.62 & 24.83 & 17.75 & 8.30 & 12.59 & 17.92 & 7.72 & 13.31 & 18.12 & 8.35 \\
\hline
\end{tabular}

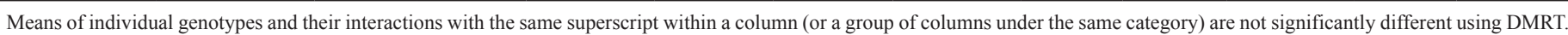
$\mathrm{NS}=$ Not significant in the footnotes.

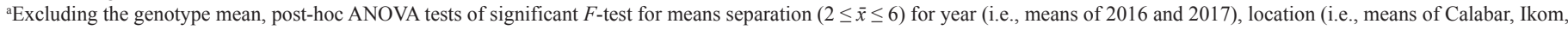

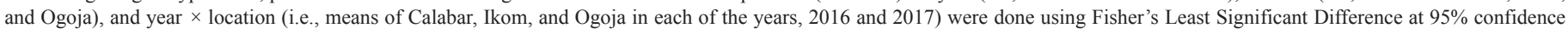
level $\left(\mathrm{LSD}_{0.05}\right)$.

* Significant at $p<0.05$

$* *$ Significant at $p<0.01$.

\subsubsection{Number of rhizome fingers}

The number of rhizome fingers per plant was significantly affected by genotype, location, location by genotype interaction, year by genotype interaction, and year by location by genotype interaction $(p<0.01)$ except for the year effect $(p>0.05)$ (Table 10). The highest number of rhizome fingers was produced in Ogoja followed by Ikom, while Calabar had the least rhizome fingers. Consequently, there was a progressive increase in the mean number of rhizome fingers across the locations from year 2016 to year 2017.

\subsubsection{Rhizome length}

Significant differences in rhizome length were recorded for genotype $(p<0.01)$, location $(p<0.01)$, year $(p<0.05)$, year by genotype interaction $(p<0.01)$, location by genotype $(p$ $<0.01$ ), and year by location by genotype $(p<0.01)$ (Table 11). The mean rhizome length of the ginger lines planted in the three locations was significantly $(p<0.05)$ different from each other with Ogoja producing the longest rhizomes followed by Ikom, while Calabar produced the shortest rhizomes. The mean rhizome length of 2017 was significantly ( $p<0.05$ ) longer than those of 2016.

\subsubsection{Rhizome yield}

Combined ANOVA showed that there were significant differences in the yield of the ginger lines as influenced by the genotype, location, location by genotype interaction, year, year by genotype interaction, and year by location by genotype interaction $(p<$ 0.01 ) (Table 12). Year had no significant effect on the yield of the ginger lines $(p>0.05)$. Among the genotypes, the highest yield was obtained from UG1-5-04 followed by UG1-5-35 and UG29-01, while the lowest yield was reported for UG1-11-07. The rhizome yield of UG1 and UG2 was not significantly ( $p>0.05)$ different. Seven out of the thirteen mutant lines derived from UG1 gave significantly $(p<0.05)$ higher yields than UG1. The two mutant lines derived from UG2 gave significantly higher yields compared to UG2. Overall, the mean yield obtained in Ogoja was not significantly $(p>0.05)$ different from that obtained in Ikom, but both yields were significantly $(p<0.05)$ higher than the yield obtained in Calabar. 
Table 7. Mean number of leaves per plant of 17 ginger lines in Calabar, Ikom, and Ogoja (2016 and 2017 early season plantings).

\begin{tabular}{|c|c|c|c|c|c|c|c|c|c|c|c|c|}
\hline \multirow{4}{*}{ Ginger line } & \multirow{4}{*}{ Genotype** } & \multirow{2}{*}{\multicolumn{2}{|c|}{$\begin{array}{c}\text { Genotype } \times \text { year }^{*} \\
\text { Year }^{\mathrm{NS}}\end{array}$}} & \multirow{2}{*}{\multicolumn{3}{|c|}{$\begin{array}{c}\text { Genotype } \times \text { location** } \\
\text { Location* }\end{array}$}} & \multirow{2}{*}{\multicolumn{6}{|c|}{$\begin{array}{c}\text { Genotype } \times \text { year } \times \text { location }^{\mathrm{NS}} \\
\text { Year } \times \text { location }^{\mathrm{NS}}\end{array}$}} \\
\hline & & & & & & & & & & & & \\
\hline & & \multirow{2}{*}{2016} & \multirow{2}{*}{2017} & \multirow{2}{*}{ Calabar } & \multirow{2}{*}{ Ikom } & \multirow{2}{*}{ Ogoja } & \multicolumn{3}{|c|}{2016} & \multicolumn{3}{|c|}{2017} \\
\hline & & & & & & & Calabar & Ikom & Ogoja & Calabar & Ikom & Ogoja \\
\hline UG1 & $16.61^{\mathrm{abc}}$ & $14.78^{\mathrm{fgh}}$ & $18.44^{\mathrm{a}-\mathrm{d}}$ & $16.83^{\mathrm{c}-\mathrm{i}}$ & $18.50^{\mathrm{a}-\mathrm{f}}$ & $14.50^{\mathrm{f}-1}$ & $13.67^{\mathrm{a}}$ & $17.33^{\mathrm{a}}$ & $13.33^{\mathrm{a}}$ & $20.00^{\mathrm{a}}$ & $19.67^{\mathrm{a}}$ & $15.67^{\mathrm{a}}$ \\
\hline UG1-11-07 & $15.44^{\mathrm{bc}}$ & $15.67^{\mathrm{c}-\mathrm{h}}$ & $15.22^{\mathrm{d}-\mathrm{h}}$ & $14.83^{\mathrm{f}-1}$ & $18.17^{\mathrm{b}-\mathrm{g}}$ & $13.33^{\mathrm{h}-1}$ & $14.33^{\mathrm{a}}$ & $17.67^{\mathrm{a}}$ & $15.00^{\mathrm{a}}$ & $15.33^{\mathrm{a}}$ & $18.67^{\mathrm{a}}$ & $11.67^{\mathrm{a}}$ \\
\hline UG1-13-02 & $18.17^{\mathrm{a}}$ & $16.89^{\mathrm{a}-\mathrm{h}}$ & $19.44^{\mathrm{ab}}$ & $16.00^{\mathrm{d}-\mathrm{k}}$ & $20.67^{\mathrm{abc}}$ & $17.83^{\mathrm{b}-\mathrm{g}}$ & $12.67^{\mathrm{a}}$ & $20.33^{\mathrm{a}}$ & $17.67^{\mathrm{a}}$ & $19.33^{\mathrm{a}}$ & $21.00^{\mathrm{a}}$ & $18.00^{\mathrm{a}}$ \\
\hline UG1-2-35 & $15.67^{\mathrm{bc}}$ & $15.22^{\mathrm{d}-\mathrm{h}}$ & $16.11^{\mathrm{c}-\mathrm{h}}$ & $15.33^{\mathrm{e}-\mathrm{k}}$ & $17.00^{\mathrm{c}-\mathrm{i}}$ & $14.67^{\mathrm{f}-1}$ & $14.67^{\mathrm{a}}$ & $17.00^{\mathrm{a}}$ & $14.00^{\mathrm{a}}$ & $16.00^{\mathrm{a}}$ & $17.00^{\mathrm{a}}$ & $15.33^{\mathrm{a}}$ \\
\hline UG1-5-04 & $15.17^{\mathrm{c}}$ & $14.00^{\mathrm{gh}}$ & $16.33^{\mathrm{b}-\mathrm{h}}$ & $17.00^{\mathrm{c}-\mathrm{i}}$ & $16.17^{\mathrm{d}-\mathrm{k}}$ & $12.33^{\mathrm{kl}}$ & $14.00^{\mathrm{a}}$ & $15.00^{\mathrm{a}}$ & $13.00^{\mathrm{a}}$ & $20.00^{\mathrm{a}}$ & $17.33^{\mathrm{a}}$ & $11.67^{\mathrm{a}}$ \\
\hline UG1-5-18 & $16.06^{\mathrm{abc}}$ & $13.67^{\mathrm{h}}$ & $18.44^{\mathrm{a}-\mathrm{d}}$ & $12.67^{\mathrm{jkl}}$ & $18.17^{\mathrm{b}-\mathrm{g}}$ & $17.33^{\mathrm{c}-\mathrm{h}}$ & $8.00^{\mathrm{a}}$ & $16.33^{\mathrm{a}}$ & $16.67^{\mathrm{a}}$ & $17.33^{\mathrm{a}}$ & $20.00^{\mathrm{a}}$ & $18.00^{\mathrm{a}}$ \\
\hline UG1-5-22 & $17.50^{\mathrm{ab}}$ & $15.00^{\mathrm{e}-\mathrm{h}}$ & $20.00^{\mathrm{a}}$ & $19.83^{\mathrm{a}-\mathrm{d}}$ & $16.17^{\mathrm{d}-\mathrm{k}}$ & $16.50^{\mathrm{d}-\mathrm{j}}$ & $14.33^{\mathrm{a}}$ & $14.67^{\mathrm{a}}$ & $16.00^{\mathrm{a}}$ & $25.33^{\mathrm{a}}$ & $17.67^{\mathrm{a}}$ & $17.00^{\mathrm{a}}$ \\
\hline UG1-5-31 & $15.22^{\mathrm{c}}$ & $15.22^{\mathrm{d}-\mathrm{h}}$ & $15.22^{\mathrm{d}-\mathrm{h}}$ & $15.33^{\mathrm{e}-\mathrm{k}}$ & $14.33^{\mathrm{g}-1}$ & $16.00^{\mathrm{d}-\mathrm{k}}$ & $15.67^{\mathrm{a}}$ & $14.00^{\mathrm{a}}$ & $16.00^{\mathrm{a}}$ & $15.00^{\mathrm{a}}$ & $14.67^{\mathrm{a}}$ & $16.00^{\mathrm{a}}$ \\
\hline UG1-5-35 & $15.44^{\mathrm{bc}}$ & $15.11^{\mathrm{e}-\mathrm{h}}$ & $15.78^{\mathrm{c}-\mathrm{h}}$ & $16.83^{\mathrm{c}-\mathrm{i}}$ & $14.67^{\mathrm{f}-1}$ & $14.83^{\mathrm{f}-1}$ & $16.00^{\mathrm{a}}$ & $15.00^{\mathrm{a}}$ & $14.33^{\mathrm{a}}$ & $17.67^{\mathrm{a}}$ & $14.33^{\mathrm{a}}$ & $15.33^{\mathrm{a}}$ \\
\hline UG1-5-38 & $16.94^{\mathrm{abc}}$ & $15.78^{\mathrm{c}-\mathrm{h}}$ & $18.11^{\mathrm{a}-\mathrm{e}}$ & $14.83^{\mathrm{f}-1}$ & $18.00^{\mathrm{b}-\mathrm{g}}$ & $18.00^{\mathrm{b}-\mathrm{g}}$ & $14.00^{\mathrm{a}}$ & $16.67^{\mathrm{a}}$ & $16.67^{\mathrm{a}}$ & $15.67^{\mathrm{a}}$ & $19.33^{\mathrm{a}}$ & $19.33^{\mathrm{a}}$ \\
\hline UG1-5-48 & $16.56^{\mathrm{abc}}$ & $15.44^{\mathrm{c}-\mathrm{h}}$ & $17.67^{\mathrm{a}-\mathrm{f}}$ & $16.33^{\mathrm{d}-\mathrm{k}}$ & $22.17^{\mathrm{a}}$ & $11.17^{1}$ & $13.33^{\mathrm{a}}$ & $21.33^{\mathrm{a}}$ & $11.67^{\mathrm{a}}$ & $19.33^{\mathrm{a}}$ & $20.00^{\mathrm{a}}$ & $10.67^{\mathrm{a}}$ \\
\hline UG1-5-49 & $15.94^{\mathrm{bc}}$ & $14.22^{\mathrm{gh}}$ & $17.67^{\mathrm{a}-\mathrm{f}}$ & $16.00^{\mathrm{d}-\mathrm{k}}$ & $16.83^{\mathrm{c}-\mathrm{i}}$ & $15.00^{\mathrm{e}-1}$ & $12.67^{\mathrm{a}}$ & $16.33^{\mathrm{a}}$ & $13.67^{\mathrm{a}}$ & $19.33^{\mathrm{a}}$ & $17.33^{\mathrm{a}}$ & $16.33^{\mathrm{a}}$ \\
\hline UG1-5-52 & $16.22^{\mathrm{abc}}$ & $15.22^{\mathrm{d}-\mathrm{h}}$ & $17.22^{\mathrm{a}-\mathrm{g}}$ & $16.17^{\mathrm{d}-\mathrm{k}}$ & $19.00^{\mathrm{a}-\mathrm{e}}$ & $13.50^{\mathrm{h}-1}$ & $14.33^{\mathrm{a}}$ & $17.67^{\mathrm{a}}$ & $13.67^{\mathrm{a}}$ & $18.00^{\mathrm{a}}$ & $20.33^{\mathrm{a}}$ & $13.33^{\mathrm{a}}$ \\
\hline UG1-7-24 & $15.72^{\mathrm{bc}}$ & $15.00^{\mathrm{e}-\mathrm{h}}$ & $16.44^{\mathrm{b}-\mathrm{h}}$ & $16.00^{\mathrm{d}-\mathrm{k}}$ & $18.17^{\mathrm{b}-\mathrm{g}}$ & $13.00^{\mathrm{i}-1}$ & $13.67^{\mathrm{a}}$ & $18.33^{\mathrm{a}}$ & $13.00^{\mathrm{a}}$ & $18.33^{\mathrm{a}}$ & $18.00^{\mathrm{a}}$ & $13.00^{\mathrm{a}}$ \\
\hline UG2 & $15.83^{\mathrm{bc}}$ & $15.22^{\mathrm{d}-\mathrm{h}}$ & $16.44^{\mathrm{b}-\mathrm{h}}$ & $12.67^{\mathrm{jkl}}$ & $21.33^{\mathrm{ab}}$ & $13.50^{\mathrm{h}-1}$ & $12.67^{\mathrm{a}}$ & $20.00^{\mathrm{a}}$ & $13.00^{\mathrm{a}}$ & $12.67^{\mathrm{a}}$ & $22.67^{\mathrm{a}}$ & $14.00^{\mathrm{a}}$ \\
\hline UG2-11-03 & $17.61^{\mathrm{ab}}$ & $16.56^{\mathrm{b}-\mathrm{h}}$ & $18.67^{\mathrm{abc}}$ & $17.67^{\mathrm{b}-\mathrm{g}}$ & $19.83^{\mathrm{a}-\mathrm{d}}$ & $15.33^{\mathrm{e}-\mathrm{k}}$ & $15.00^{\mathrm{a}}$ & $18.67^{\mathrm{a}}$ & $16.00^{\mathrm{a}}$ & $20.33^{\mathrm{a}}$ & $21.00^{\mathrm{a}}$ & $14.67^{\mathrm{a}}$ \\
\hline UG2-9-01 & $16.83^{\mathrm{abc}}$ & $17.00^{\mathrm{a}-\mathrm{g}}$ & $16.67^{\mathrm{b}-\mathrm{h}}$ & $15.67^{\mathrm{e}-\mathrm{k}}$ & $18.33^{\mathrm{b}-\mathrm{g}}$ & $16.50^{\mathrm{d}-\mathrm{j}}$ & $14.67^{\mathrm{a}}$ & $19.33^{\mathrm{a}}$ & $17.00^{\mathrm{a}}$ & $16.67^{\mathrm{a}}$ & $17.33^{\mathrm{a}}$ & $16.00^{\mathrm{a}}$ \\
\hline Mean $^{\mathrm{a}}$ & 16.26 & 15.30 & 17.23 & 15.88 & 18.00 & 14.90 & 13.75 & 17.39 & 14.75 & 18.02 & 18.78 & 15.06 \\
\hline SEM & 0.281 & 0.34 & 0.41 & 0.55 & 0.38 & 0.36 & 0.43 & 0.51 & 0.43 & 0.69 & 0.54 & 0.59 \\
\hline CV (\%) & 17.42 & 15.76 & 16.92 & 20.03 & 12.32 & 14.03 & 12.85 & 12.08 & 11.92 & 15.75 & 11.94 & 16.09 \\
\hline
\end{tabular}

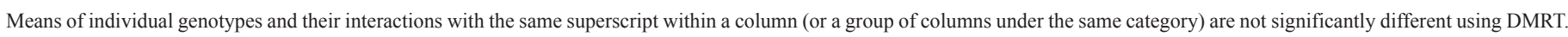
$\mathrm{NS}=$ Not significant in the footnotes.

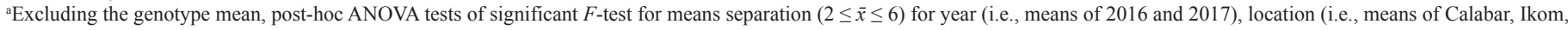

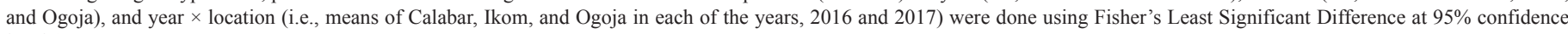
level $\left(\mathrm{LSD}_{005}\right)$.

* Significant at $p<0.05$.

** Significant at $p<0.01$.

\section{DISCUSSION}

In the present study, it is established that the variation in genotypes was highly significant for all the measured traits except establishment count. The ginger mutant lines were significantly different $(p<0.05 ; p<0.01)$ in their mean performances for sprouting percentage, plant height, number of leaves per plant, leaf length, leaf width, leaf area, number of tillers per plant, number of rhizome fingers per plant, rhizome length, and rhizome yield. These significant variations in the phenotypic expression of the various characteristics might be due to the inherent genetic properties of the ginger mutant lines; this reveals the presence of considerable genetic variations in the ginger mutant lines for these attributes [13]. It also implies the potentials of these mutant lines to be used as source material for the genetic improvement of growth and yield characters in ginger in these areas. According to Sumanth et al. [14], the success of any crop improvement programme depends on the extent of genetic variations that exist in the available germplasm of that crop. Ginger lines used in this study were inconsistent in their performances across the different characters. Similar significant variations among ginger genotypes were also observed by Goudar et al. [15], Jatoi and Watanabe [13], and Aragaw et al. [16] who reported significant genotype differences across several growth and yield characters in ginger. The superior performance in yield and other characters in some of the mutant lines over the check landraces from which they were derived showed that the variability created by mutation had positive effects on the mutant lines. Improvements in the growth and yield traits of different crops through mutation breeding have been reported by several crop breeders [4,17-19]. Inducing mutation has been considered an established method for increasing genetic variability in many crops. Mutation breeding is an essential tool in crop improvement of vegetatively propagated crops, especially in plants with reproductive sterility. According to Food and Agriculture Organization of the United Nations [20], over 2,450 varieties in different crops developed through mutation breeding have been commercially released. This includes vegetatively propagated crops such as garlic, cassava, turmeric, and potato. Breeding of ginger is greatly challenged by poor flowering and seed set and a great number of crop improvement programmes of these genotypes are limited to evaluation and selection of naturally 


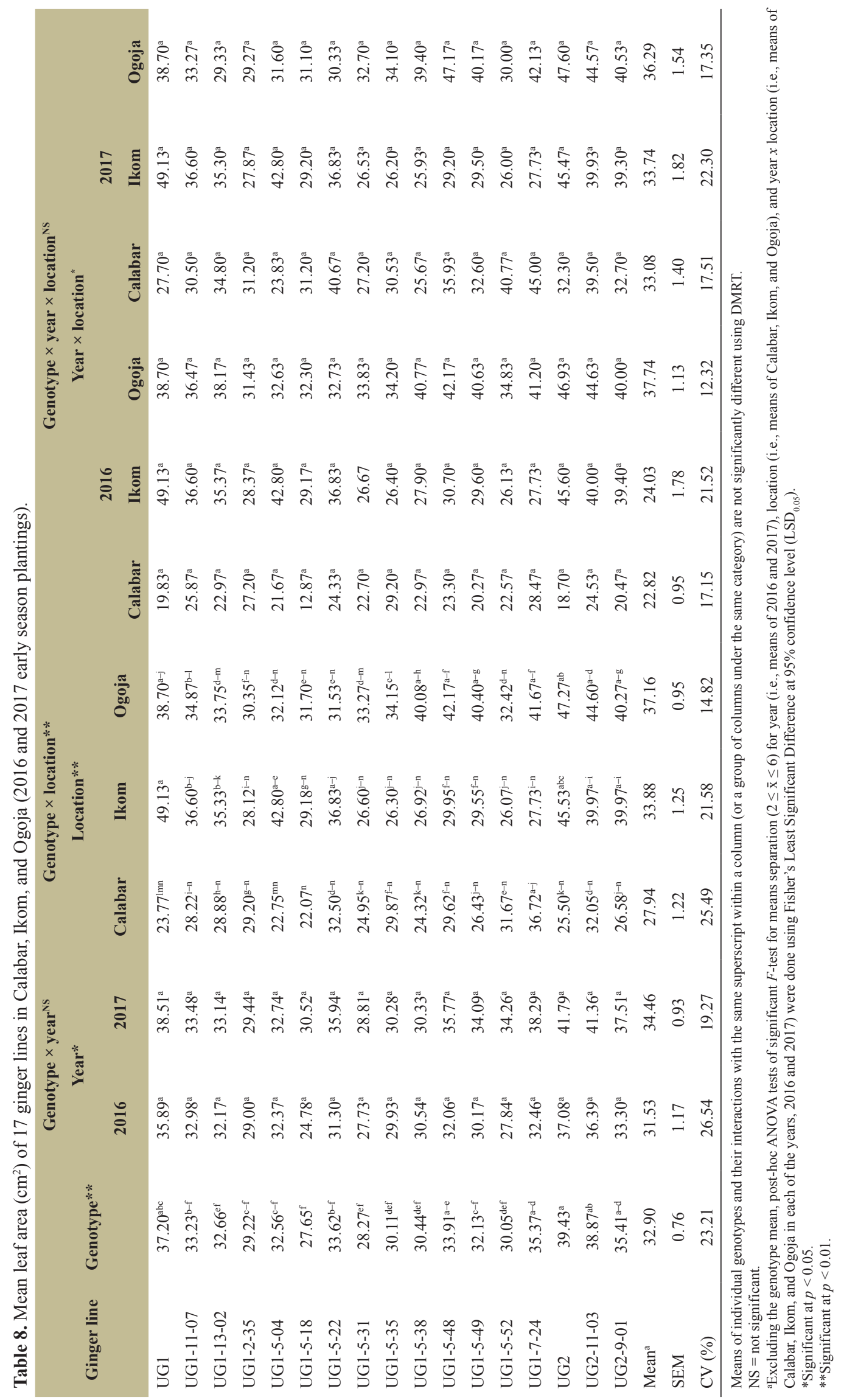




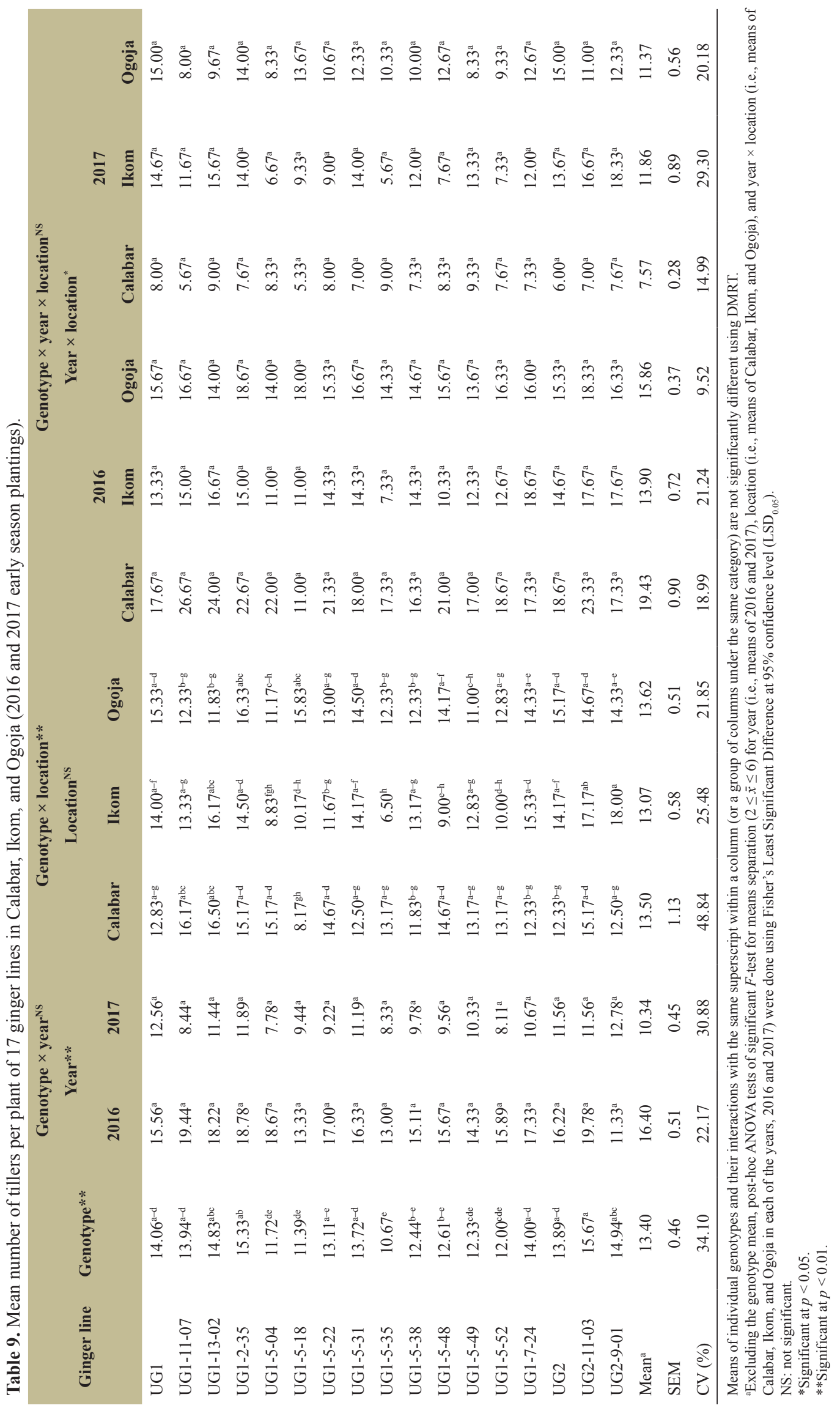




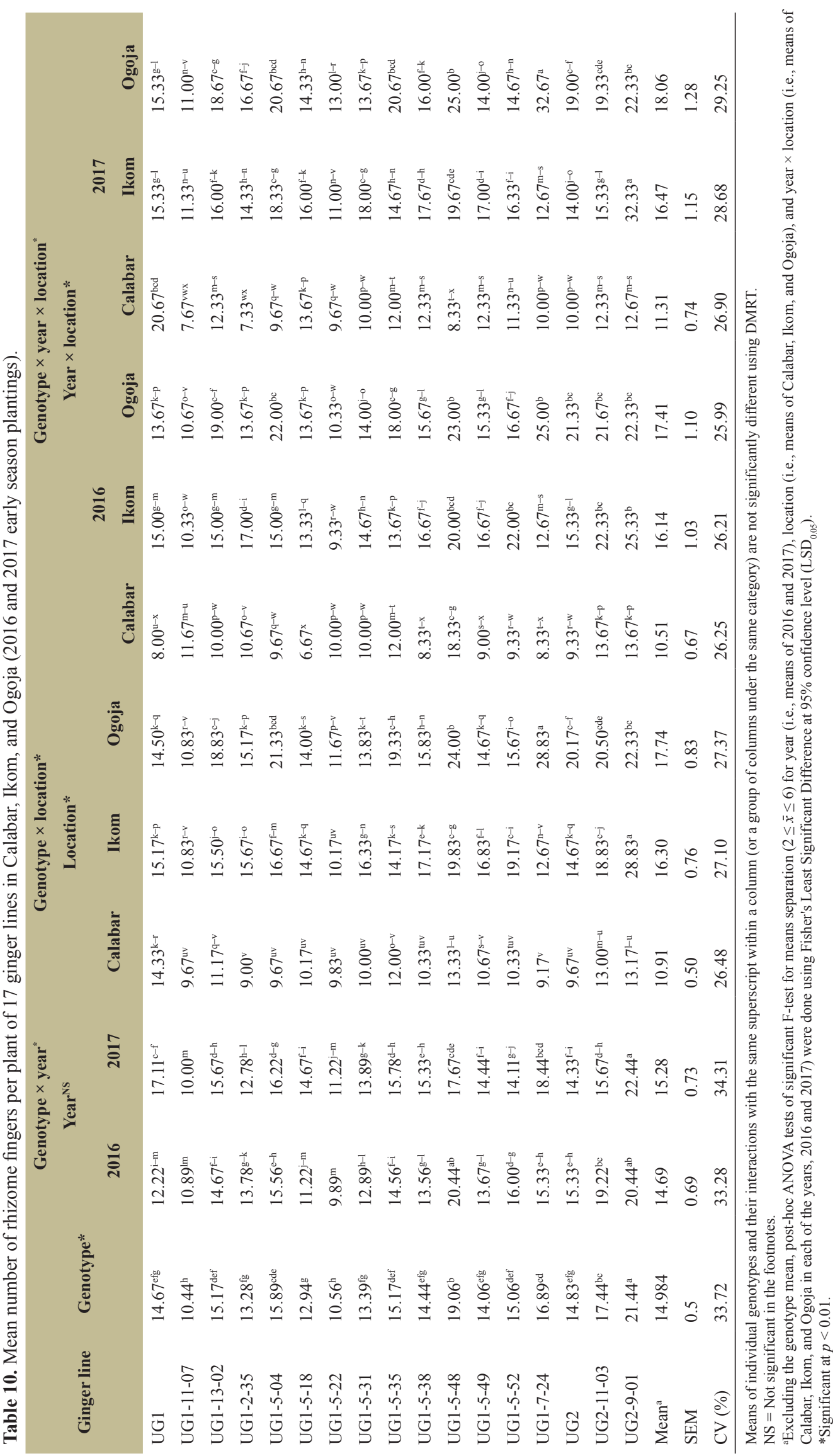




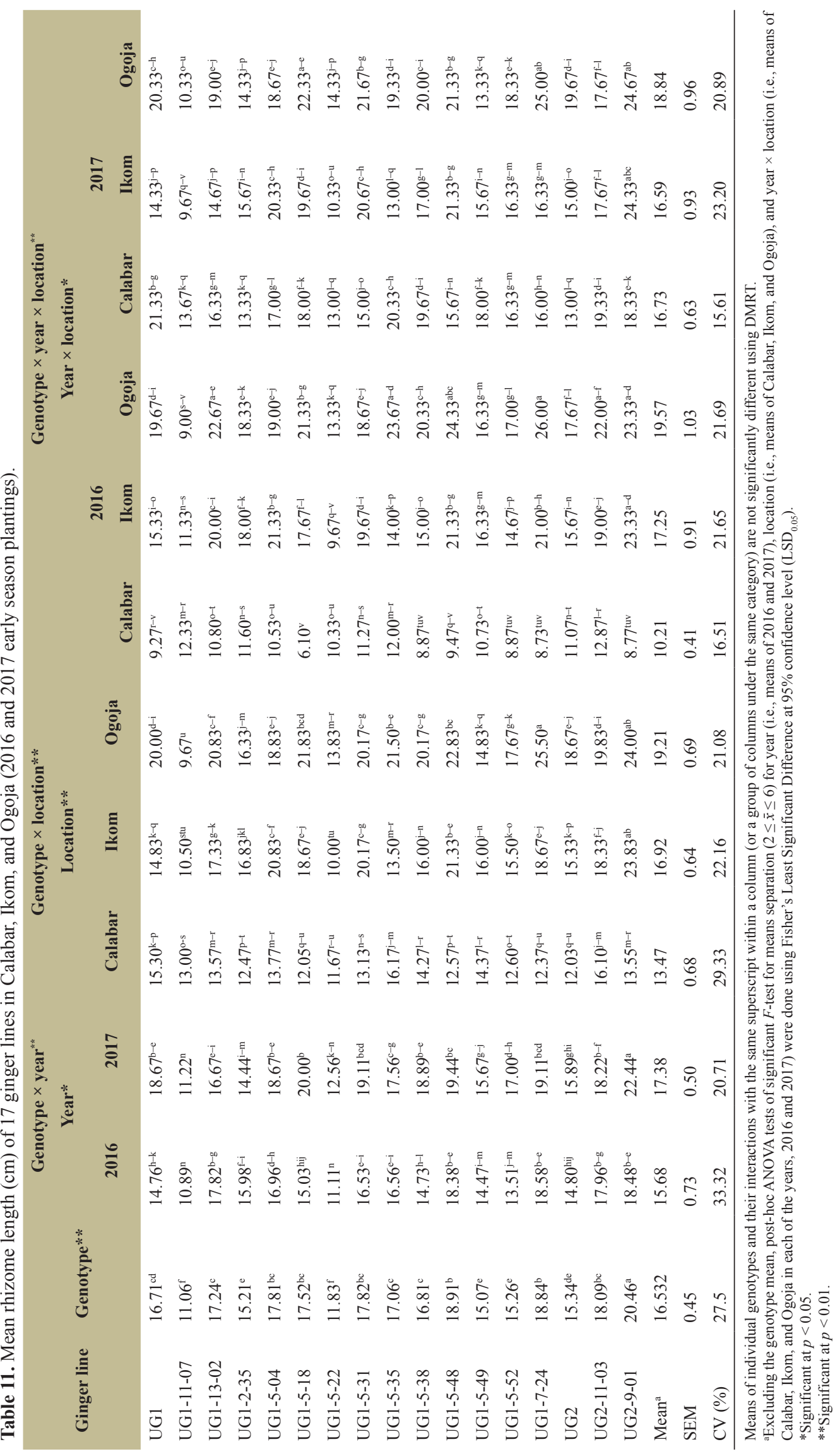




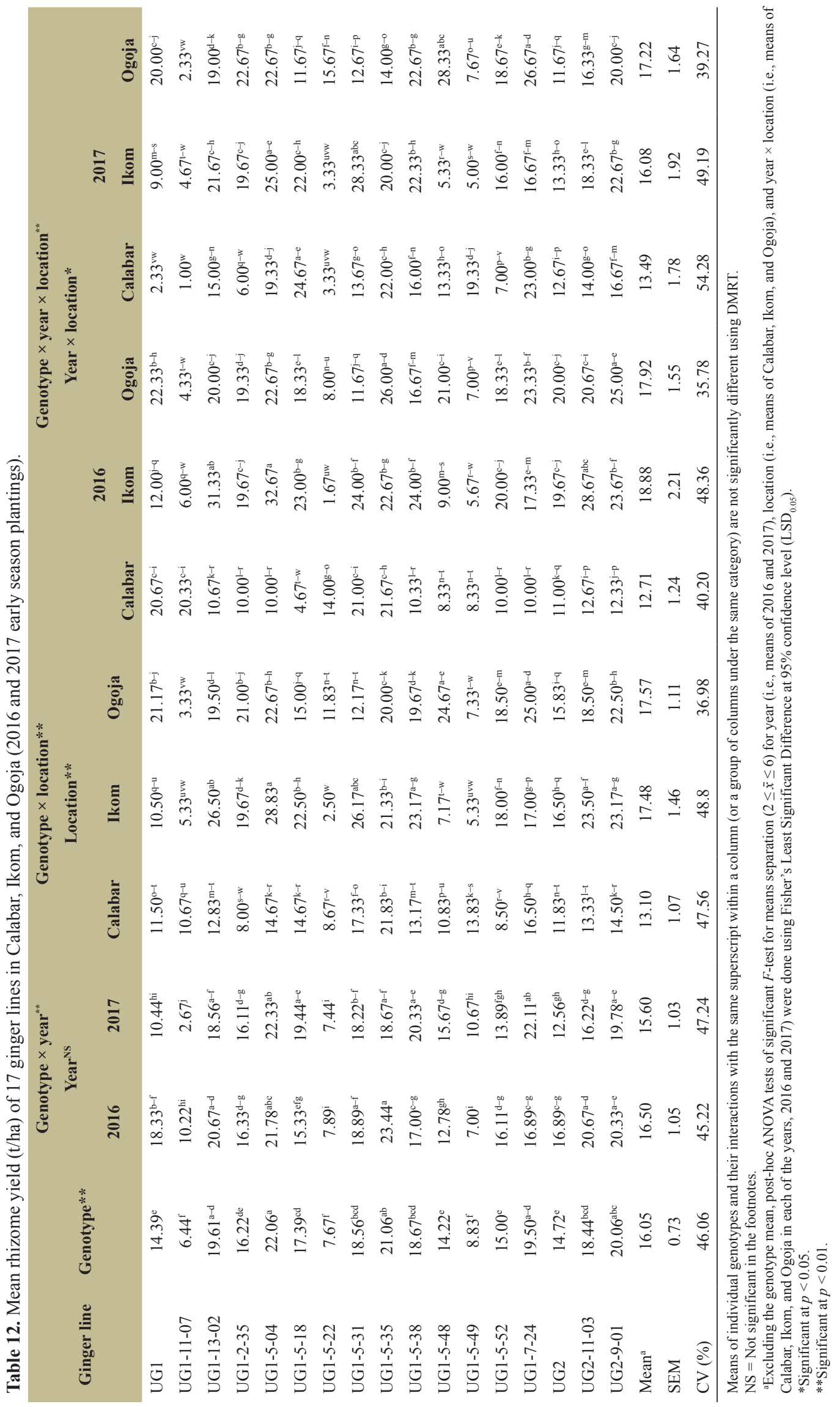


occurring variations [21]. Induced mutation is therefore an alternative and reliable way of creating variability in these crops [18]. Hence, the observations recorded in the yield performances of the mutant lines used in this study have significant implications. The results also showed that location significantly affected the growth and the yield characters of the ginger lines.

The variation in yield in Ogoja and Ikom was not significantly different but was significantly higher than those of Calabar. This result suggests Ogoja and Ikom as favorable locations for ginger cultivation. Attoe et al. [22] also observed significant differences in the growth and yield parameters of ginger genotypes across soils of different locations in Cross River State. The high significant effects of genotype by environment interaction observed for yield and most of the traits clearly demonstrate that genotype and environment interaction across the environments play an important role in breeding adaptable genotypes to wide environments. The variations observed in yield and yield traits of the ginger lines across the locations and years may be attributed to the inherent transferable parental trait differences in the ginger lines as well as the environmental influence. Mohandas et al. [23] also observed significant genotype by environment interactions in some growth and yield traits of ginger. Alghamdi [24] reported that significant genotype by environment interactions suggests that, across environments, some genotypes were more stable than others. According to Ghaffari and Depao [25], yield differences attributed to environment are relevant to genotype evaluation and mega-environment investigations.

\section{CONCLUSION}

UG2-11-03 gave superior performances for plant height and number of tillers per plant. Its performances across the other traits were relatively high and above average for most. UG2-9-01 had the highest number of rhizomes per plant and also produced the longest rhizomes. With respect to rhizome yield, UG1-5-04 was the highest performing mutant line followed by UG1-5-35 and UG2-9-01. Ginger rhizome yields in Ogoja and Ikom were not significantly different from each other but both were significantly higher than that obtained in Calabar. The locations Ikom and Ogoja are recommended as good environments for ginger cultivation. The information obtained from this research work will be important in the development of excellent selection procedures for the improvement of ginger genotypes under this agroecology and also serve as a source of useful information for the cultivation of ginger in the region. However, further evaluation and testing of these ginger mutant lines in other agroecological zones and across different environments will be necessary before subsequent release.

\section{ACKNOWLEDGMENTS}

The authors wish to acknowledge the Ginger Research Unit of the NRCRI Umudike, Abia State, Nigeria, for making the ginger lines used for this research available.

\section{CONFLICT OF INTEREST}

The authors declared that there are no conflicts of interest.

\section{AUTHOR CONTRIBUTIONS}

All authors made substantial contributions to conception and design, acquisition of data, or analysis and interpretation of data; took part in drafting the article or revising it critically for important intellectual content; agreed to submit to the current journal; gave final approval of the version to be published; and agree to be accountable for all aspects of the work. All the authors are eligible to be an author as per the international committee of medical journal editors (ICMJE) requirements/guidelines.

\section{FUNDING}

There is no funding to report.

\section{ETHICAL APPROVALS}

This study does not involve experiments on animals or human subjects.

\section{PUBLISHER'S NOTE}

This journal remains neutral with regard to jurisdictional claims in published institutional affiliation.

\section{REFERENCES}

1. Kizhakkayil J, Bhas S. Diversity, characterization and utilization of ginger: a review. Plant Genet Resour 2011;9:464-77; doi:10.1017/ S1479262111000670

2. Famurewa AV, Emuekele PO, Jaiyeoba KF. Effect of drying and size reduction on the chemical and volatile oil content of ginger. J Med Plants Res 2011;5(14):2941-4; doi:10.5897/JMPR.9000476

3. Kalaivani K, Senthil N, Murugesan, GA. Biological activities of selected Lamiaceae and Zingiberaceae. Parasitol Res 2012;11(3):1261-8; doi:10.1007/s00436-011-2623-x

4. Sarwar A, Butt SJ. Evaluation of mutant lines of Rosa species. Adv Crop Sci Technol 2016;3(5):1-5; doi:10.4172/2329-8863.1000196

5. Amadi CO. (2012). Ginger breeding in Nigeria: challenges and prospect. J Appl Agric Res 2012;4(2):155-63.

6. Food and Agriculture Organization of the United Nations. Production quantity of ginger in metric tonnes. Food and Agriculture Organization of the United Nations, Rome, Italy, 2018.

7. Ezeagu W. Ginger export. A paper presented at 3-day National Workshop on massive cassava and ginger production and processing for local industries and export, held at Fati Muasu Hall. National Centre for Women development, Abuja, Nigeria, 2006.

8. Iwo GA, Ekaette EA. Genetic components analysis of yield related traits in some ginger genotypes. Niger J Genet 2010;23:81-5.

9. Nmadu JN, Marcus PL. Efficiency of ginger production in selected Local Government of Kaduna State, Nigeria. Int J Food Agric Econ 2013;1(2):39-52. Available via http://www.foodandagriculturejournal. com/Vol\%201\%20No\%202\%2039-52.pdf

10. Chukuwu GO, Emehuite JK. Fertilizer efficiency and productivity of ginger on a hapilyariscol in southern Nigeria. In: Akoroda MO (ed.). Root crops: the small processor and development of local food industries for market economy. Ibadan Polytechnic Venture, Ibadan, Nigeria, 2003.

11. Effiong J. Changing the pattern of use in the Calabar river catchment South eastern Nigeria. J Sustain Dev 2011;4(1):92-102; doi:10.5539/ jsd.v4n1p92

12. Gomez KA, Gomez AA. Statistical procedures for agricultural research. 2nd edition, John Wiley \& Sons, New York, NY, p 680, 1984.

13. Jatoi AS, Watanabe, KN. Diversity analysis and relationship among ginger landraces. Pak J Bot 2013;45(4):1203-14. Available via http:// pakbs.org/pjbot/ 


$$
\text { 2021;9(05):110-123 }
$$

14. Sumanth V, Suresh BG, Ram BJ, Srujana G. Estimation of genetic variability, heritability, and genetic advance for grain yield components in rice (Oryza sativa L.). J Pharmacol Phytochemistry 2017;6(4):1437-9. Available via https:/www.phytojournal.com/ archives/2017/vol6issue4/PartU/6-4-59-298.pdf

15. Goudar SA, Gangadharappa PM, Dodamani SM, Lokesh C, Dharamatti VU. Evaluation of ginger (Zingiber officinale) genotypes for growth and yield attributes. Int J Pure Appl Biosci 2017;5(2):994 9; doi:10.18782/2320-7051.2888

16. Aragaw MS, Alamerew G, Michael H, Tesfaye A. Variability of ginger (Zingiber officinale Rosc.) accessions for morphological and some quality traits in Ethiopia. Int J Agric Sci 2011;6:444-57; doi:10.3923/ ijar.2011.444.457

17. Edirimanna EP, Korla BN. Induced variation for yield and quality characters of ginger (Zingiber officinale) using ethyl methane sulphonate. Ann Sri Lanka Dep Agric 2007;9:9-17.

18. Selvarasu A, Kandhasamy R. Analysis of variability, correlation and path coefficient in induced mutants of glory lily (Gloriosa superb L.). Int J Plant Breed 2013;7(1):69-75. Available via http://www. globalsciencebooks.info/Online/GSBOnline/images/2013/IJPB 7(1)/ IJPB 7(1)69-75o.pdf

19. Raina A, Lasker RA, Khursheed S, Amin R., Tantray YR, Parveen $\mathrm{K}$, et al. Role of mutation breeding in crop improvement - past, present and future. Asian Res J Agric 2016;2(2):1-13; doi:10.9734/ ARJA/2016/29334

20. Food and Agriculture Organization of the United Nations. Ginger: post-production management for improved market access. Prepared by Plotto, A. Edited by Mazaud, F, Rotter, A and Steffel, K. Food and Agriculture Organization of the United Nations, Rome, Italy, 2006.

21. Givilidharan MP, Balakrishnan S. Gamma ray induced variability in vegetative and floral characters of ginger. Indian Cocoa, Arecanut Spices J 1992;15:68-72.
22. Attoe EE, Ojikpong TO, Kekong MA. Evaluation of growth and yield parameters of two ginger varieties in different soils of Cross Rivers State, Nigeria. Eur J Acad Essays 2016;3(3):109-20.

23. Mohandas, TP, Pradeep Kumar, T, Mayadevi, P, Aipe, KC, Kumaran, K. Stability analysis in ginger (Zingiber officinale Rosc.) genotypes. J Spices Aromat Crops 2000;9:165-7; http://updatepublishing.com/ journal/index.php/josac/article/view/4574

24. Alghamdi SS. Yield stability of some soybean genotypes across diverse environments. Pak J Biol Sci 2004;7(12):2109-14; doi:10.3923/ pjbs.2004.2115.2120

25. Ghaffari AA, Depao E. Agroclimatic zoning of Iranian cold drylands. In the Proceeding of the Iranian Crop Sciences Congress, Karaj, Iran, 2006, pp 42-48.

\section{How to cite this article:}

Abua MN, Iwo GA, Ittah MA, Obok EE, Edugbo RE. Growth and yield performance of mutant ginger (Zingiber officinale Rosc.) lines in South-Eastern Nigeria. J Appl Biol Biotech 2021; 9(05):110-123. 